\title{
1 c-Myc plays a key role in IFN-y induced persistence of Chlamydia trachomatis
}

2 Nadine Vollmuth ${ }^{1}$, Sudha Janaki-Raman², Lisa Schlicker ${ }^{\#}$, Naziia Kurmasheva ${ }^{1}$, Werner

3 Schmitz ${ }^{2}$, Almut Schulze ${ }^{2, \#}$, Thomas Rudel $^{1^{*}}$ and Karthika Rajeeve ${ }^{1, \S}$

$4{ }^{1}$ Department of Microbiology, Biocenter, University of Wuerzburg, Biocenter, Am Hubland, D-97074 Wuerzburg, 5 Germany.

$6{ }^{2}$ Department of Biochemistry and Molecular Biology, University of Wuerzburg, Biocenter, Am Hubland, D-97074

7 Würzburg, Germany

$8 \quad$ \# German Cancer Research Center (DKFZ), Im Neuenheimer Feld 581, 69120 Heidelberg, Germany.

$9 \S$ Department of Biomedicine, University of Aarhus, The Skou Building, Høegh-Guldbergs Gade 10, DK-8000,

10 Aarhus C, Denmark.

11 * Correspondence: thomas.rudel@biozentrum.uni-wuerzburg.de

\section{Summary}

Chlamydia trachomatis (Ctr) can persist over long periods of time within their host cell and thereby establish chronic infections. One of the major inducers of chlamydial persistence is interferon-gamma (IFN- $\gamma$ ) released by immune cells as a mechanism of immune defence. IFN-y activates the catabolic depletion of L-tryptophan (Trp) via indoleamine 2,3-dioxygenase (IDO), resulting in persistent Chlamydia. Here we show that IFN- $\gamma$ depletes c-Myc, the key regulator of host cell metabolism, in a STAT1-dependent manner. Expression of c-Myc rescued Chlamydia from IFN-y-induced persistence in cultured cell lines, but also in human fallopian tube organoids. L-tryptophan concentrations control c-Myc levels via the PI3KGSK3 $\beta$ axis. Unbiased metabolic analysis revealed that Chlamydia infection reprograms the host cell tricarboxylic acid (TCA) cycle to support pyrimidine biosynthesis. Addition of TCA cycle intermediates or pyrimidine/purine nucleosides to infected cells rescued Chlamydia from IFN- $\gamma$-induced persistence. Thus, our results challenge the longstanding hypothesis of L-tryptophan depletion through IDO as the major mechanism of IFN- $\mathrm{\gamma}$-induced metabolic immune defence and significantly extends the understanding of the role IFN- $\gamma$ as a broad modulator of host cell metabolism. 
Chlamydia trachomatis, persistence, interferon-gamma, c-Myc, TCA cycle.

\section{Introduction}

Chlamydia trachomatis (Ctr) is an obligate intracellular human pathogen, which causes a broad range of acute and chronic diseases ${ }^{1}$. It is the leading cause of bacterial sexually transmitted diseases (STD), with more than 130 million new cases annually². Infection of the urogenital tract by Chlamydia can lead to urethritis, infertility, elevated risk of foetal and neonatal deaths, ectopic pregnancies and pelvic inflammatory disease (PID) $)^{3,4}$. Furthermore, left untreated, Chlamydia infection increases the risk of HIV infections ${ }^{5,6}$ and might contribute to the development of cervical and ovarian cancer ${ }^{7-10}$. The pathogen extensively interferes with the physiology of the infected cell, since it depends entirely on its host cell as a replicative niche during infection ${ }^{11}$.

Chlamydiae have a unique biphasic life cycle, consisting of two physiologically and morphologically distinct forms ${ }^{12}$. This gram-negative bacterium initiates its developmental cycle by attachment and invasion of the host cell by the elementary body (EB), the nondividing infectious form of the pathogen. Inside the cell, EB stay within the endosomes, which they modify rapidly to create a replicative niche termed 'inclusion', to avoid lysosomal degradation $^{13}$. EB differentiate into reticulate bodies $(R B)$, which is the non-infectious replicating form of the pathogen. It takes several rounds of cell division until RB redifferentiate back into EB and the progenies are released by host cell lysis or extrusion, an exocytosis like mechanism, to infect neighbouring cells ${ }^{14,15}$. Apart from active infection, Chlamydia can turn into a dormant state called persistence and can remain over a long time, maybe even years ${ }^{16}$ within its host cell and thereby establish a chronic infection. During the persistent state, the pathogen remains viable and replicates its genome, but it exhibits decreased metabolic activity and inhibited cell division, which leads to the formation of enlarged pleomorphic aberrant bodies $(A B)^{17}$. The transition to the persistent form might represent an important chlamydial survival mechanism against antibiotics and the immune response of the host, since this process is reversible ${ }^{17,18}$. The conversion to this dormant 
57 stage is induced by penicillin ${ }^{19}$, iron deficiency ${ }^{20}$, amino acid starvation ${ }^{21}$ and interferongamma (IFN- $y)^{17,18}$. IFN- $\gamma$ is an immune regulated cytokine, which is involved in the cellintrinsic immunity against several intracellular pathogens, including Chlamydia ${ }^{22}$. The downstream signalling pathways activated by IFN- $y$ are highly species specific and differ dramatically between mouse and human cells ${ }^{23}$. In human cells, the anti-chlamydial effect of IFN-y is predominantly mediated by the induction of indoleamine-2,3-dioxygenase (IDO), an enzyme that catalyses the initial step of L-tryptophan (Trp) degradation to $\mathrm{N}$-formyl kynurenine and kynurenine ${ }^{24}$. Trp is a crucial amino acid for chlamydial development ${ }^{25,26}$, and its depletion leads to persistence ${ }^{18,27}$.

IFN-y belongs to the type II interferons that bind to the extracellular domain of the interferongamma receptor, which is a heterodimer of the two subunits IFNGR1 and IFNGR2. The intracellular domains of the IFNGR1 subunits are associated with Janus kinase 1 (Jak1), while the IFNGR2 subunits are associated with Jak2. Activation of Jak1 and Jak2 results in phosphorylation of the receptor and subsequent recruitment and phosphorylation of signal transducer and activator of transcription (STAT1). STAT1 phosphorylation at tyrosine 701 and serine 727 leads to its homodimerization and nuclear translocation. Once in the nucleus, STAT1 homodimers bind to IFN- $\gamma$-activated sequence (GAS) elements in the promoters of target genes to regulate their transcription ${ }^{28-30}$. IFN- $\gamma$ can function as both, a growth inhibiting or promoting cytokine, in a STAT1-dependent manner ${ }^{31,32}$. Binding of STAT1 homodimers to the consensus GAS elements in the $c-m y c$ promoter inhibits its expression transcriptionally ${ }^{28}$. Concurrently, $c-M Y C$ expression is not only negatively regulated by STAT1 but stat 1 is also a negative target gene of c-Myc. Hence, c-Myc and STAT1 regulate each other in a negative feedback loop at the transcriptional level ${ }^{33}$.

The transcription factor c-Myc targets genes involved in the regulation of numerous cellular processes, such as cell proliferation, cell growth, translation, metabolism and apoptosis ${ }^{34-37}$. Furthermore, c-Myc activity increases energy production, anabolic metabolism, promotion of aerobic glycolysis and glutaminolysis, inducing mitochondrial biogenesis and tricarboxylic acid (TCA) cycle activity ${ }^{37}$. Glutaminolysis increases the production of biomass by providing 
TCA cycle intermediates via anaplerosis, which enhances their availability for the production of amino acids, nucleotides and lipids ${ }^{38}$. c-Myc also critically controls nucleotide biosynthesis by directly regulating the expression of genes that encode the enzymes involved in the production of precursors of all nucleotides ${ }^{39,40}$. For example, c-Myc directly controls the cisregulatory element in the 5'-UTR of phosphoribosyl pyrophosphate synthetase (PRPS), which catalyses the first committed step in purine biosynthesis ${ }^{41}$. In pyrimidine biosynthesis, the rate-limiting step is catalysed by carbamoyl aspartate dehydratase $(C A D)^{42}$, which is also regulated by $\mathrm{c}-\mathrm{Myc}$ as a response to growth stimulatory signals, such as activation of EGFR/RAS/MAP kinase ${ }^{43}$, Hif $1 / 2 a^{44}$ or estrogen receptor/Sp1 $1^{45}$ pathways. We recently identified a central role of c-Myc in the control of the metabolism in Chlamydia-infected cells $^{46}$. Chlamydia has a reduced genome and only very limited metabolic capacity. For example, they have a truncated TCA cycle and lack the ability to synthesize purine and pyrimidine nucleotides de novo but acquire ATP and nucleosides from the host cell ${ }^{47,48}$.

Expression of IDO and depletion of Trp has been previously described as the main reason for interferon-induced persistence in Chlamydia ${ }^{49,50}$. Here we show that IFN-y induces a STAT1-dependent depletion of c-Myc in Chlamydia-infected cells, which de-regulates the host metabolism and induces Chlamydia persistence. Importantly, addition of the TCA cycle intermediate $\alpha$-ketoglutarate or pyrimidine/purine nucleosides was sufficient to prevent persistence and restore chlamydial replication. Our data demonstrate a central role of c-Mycregulated metabolic pathways in the IFN- $\gamma$-induced persistence of $C$. trachomatis.

\section{Results}

IFN-y treatment prevents c-Myc induction and impairs the development of Chlamydia

c-Myc is an important regulator of host cell metabolism and is indispensable for chlamydial acute infection and progeny formation ${ }^{46}$. Since Chlamydia obtain all nutrients from the host cell and it has been shown that several environmental conditions affecting host cell 
111 metabolism like iron and amino acid starvation also induce chlamydial persistence ${ }^{51,52}$, we

112 investigated the role of c-Myc in chlamydial persistence. From our previous study, it was

113 known that ablation of c-Myc expression interfered with chlamydial development and progeny

114 formation ${ }^{46}$. To investigate if Chlamydia enter a persistence state in c-Myc depleted cells, we

115 infected a HeLa 229 cell line with an anhydrotetracycline (AHT)-inducible short hairpin RNA

116 (shRNA) for c-Myc in the absence and presence of the inducer. In agreement with our

117 previous results ${ }^{46}$, silencing of c-Myc expression prevented inclusion formation (Fig. 1a). We

118 then cultivated cells for $24 \mathrm{~h}$ with AHT to suppress c-Myc expression and then either added

119 AHT for another $12 \mathrm{~h}$ or removed AHT to re-establish c-Myc expression (see scheme Fig.

120 1a). In contrast to the cells with silenced c-Myc, removal of AHT efficiently restored inclusion

121 formation (Fig. 1a), suggesting that suppression of c-Myc induces a persistence state in

122 Chlamydia. Removal of AHT after $72 \mathrm{~h}$ still restored chlamydial inclusion formation, however,

123 the HeLa cells started to lyse due to the long cultivation time (not shown). A physiological

124 mechanism to induce chlamydial persistence is the exposure of infected cells to IFN- $\gamma$ which

125 causes the depletion of Trp via the induction of IDO ${ }^{24}$. Interestingly, IFN- $y$-treated cells failed

126 to stabilize c-Myc upon Chlamydia infection (Fig. 1b). In contrast, induction of persistence by

127 antibiotic treatment, which targets the bacterial rather than host cell metabolism, did not alter

128 c-Myc levels (Fig. 1b). Nevertheless, both modes of persistence induction caused a failure of

129 the bacteria to produce infectious progenies (Extended data Fig. 1a).

130 c-Myc protein stability is regulated by two phosphorylation sites with opposing functions.

131 Serine 62 phosphorylation (pS62) stabilizes c-Myc whereas threonine 58 phosphorylation

132 (pT58) promotes c-Myc degradation ${ }^{53}$. IFN- $y$ treatment led to decreased phosphorylation of

c-Myc at serine 62, a modification of c-Myc that prevents the ubiquitination and proteasomal 134 degradation of the protein ${ }^{54}$, while phosphorylation at threonine 58 was unchanged (Fig. 1c).

135 c-Myc was also depleted upon IFN-y treatment of primary cells from human fimbriae (Fimb),

136 although the concentration of IFN- $y$ required to achieve the same effect was five times higher

137 than in HeLa 229 cells ( $10 \mathrm{ng} / \mathrm{mL}$ in HeLa 229 compared to $50 \mathrm{ng} / \mathrm{mL}$ in Fimb) (Extended 
data Fig. 1b, c). Similar results were obtained with a serovar D strain involved in STD

139 (Extended data Fig. 1d).

140 IFN-y is known to signal via the JAK-STAT pathway via phosphorylation of STAT1 and

141 transcriptional regulation of gene expression (Fig. 1d). Treatment with IFN- $\gamma$ led to the 142 phosphorylation of STAT1 at serine 727 and tyrosine 701 (Fig. 1f) and prevented the 143 induction of c-Myc protein (Fig. 1f) and mRNA expression (Fig. 1e) upon Chlamydia infection.

144 To verify that interferon signals via STAT1 to transcriptionally deplete c-Myc, we used siRNA 145 against STAT1 (Fig. 1g). STAT1 depletion rescued c-Myc levels and chlamydial growth in 146 primary infections (Fig. 1g). When bacteria from IFN-y treated and STAT1-depleted cells 147 were transferred to fresh cells, infectious progeny could be recovered, indicating that STAT1 downregulates c-Myc and prevents chlamydial development (Extended data Fig. 1e).

The IFN-y response in humans and mice is entirely different, limiting the use of murine systems as models for human pathogenic $C$. trachomatis infections ${ }^{23}$. We therefore established an IFN-y-induced persistence model in human fallopian tube organoids. Healthy tissue obtained from patients that underwent hysterectomy was used to establish organoid cultures. These organoids obtained from five different patients were pre-treated with IFN- $y$ for $2 \mathrm{~h}$ and then infected with Chlamydia for 6 days (Fig. 2a, b). In this human infection model, IFN-y treatment strongly reduced inclusion formation (primary infection: Fig. 2b) and production of infectious progeny (Fig. 2c, d; Extended Fig. 2). Moreover, the IFN- $\mathrm{Y}$-STAT1 signalling axis was active in human organoids and efficiently prevented the induction of cMyc upon infection (Fig. 2e), confirming the physiological significance of this system as a model of persistence infection.

Expression of C-Myc rescues Chlamydia from IFN- $\gamma$-induced persistence

As we observed a key role of c-Myc in chlamydial persistence, we next asked if maintaining c-Myc levels can overcome IFN- - -induced persistence. To address this question, we used a WII-U2OS cell line in which c-Myc expression is under the control of an AHT-inducible promoter. These cells were used as infection models for IFN- $\mathrm{\gamma}$-induced persistence under 
constant c-Myc expression. Bacterial replication efficiency and the ability to produce

infectious progenies were studied. Interestingly, constant expression of c-Myc efficiently suppressed IFN- - -induced persistence (Fig. 3a) and supported the development of infectious progeny (Fig. 3b, c; Extended data Fig. 3a). We also tested the effect of c-Myc on persistence in organoids derived from human fallopian tubes. Organoids were transduced with lentivirus expressing c-Myc or GFP as control (Extended data Fig. 3b-d) and selected for puromycin resistance (Extended data Fig. 3e). These organoids were then infected with Chlamydia and treated with IFN-y. Maintaining expression of c-Myc rescued the strong suppression of infectious progeny development by IFN-Y also in fallopian tube organoids

174 (Fig. 3d, e), demonstrating that downregulation of c-Myc is essential for IFN-y-mediated persistence in this human infection model.

It has been shown previously that IFN- $\mathrm{Y}$-mediated persistence can be overcome by the addition of exogenous $\operatorname{Trp}^{18}$. To investigate the importance of $\operatorname{Trp}$ in the context of IFN- $\mathrm{Y}$ mediated regulation of c-Myc expression in our model system, HeLa 229 and human Fimb cells were treated with IFN- $v$, infected with Chlamydia and provided with exogenous Trp. Subsequently, bacterial replication efficiency as well as production of infectious progenies was analysed by Western blotting. As expected, addition of Trp rescued chlamydial growth after IFN-y treatment (Extended data Fig. 4a) and gave rise to infectious progenies (Fig. 4a; Extended data Fig. 4b). Surprisingly, addition of Trp also resulted in the stabilization of cMyc, even without chlamydial infection (Extended data Fig. 4c).

All C. trachomatis strains are Trp auxotroph but the genital strains retain an L-tryptophan synthase $(\operatorname{trp} B)$, which uses exogenous indole provided by the microflora in the genital tract as a substrate to synthesize $\operatorname{Trp}^{27,55,56}$. Thus, we validated if exogenous indole recovers chlamydial growth and leads to infectious progenies. Intriguingly, indole addition resulted not only in suppression of persistence (Extended data Fig. 4d) and formation of infectious 
192 progeny (Extended data Fig. 4e), but also in the stabilization of c-Myc in both infected and non-infected cells (Extended data Fig. 4d).

Next, we tested whether Trp alone is sufficient to support Chlamydia development also in the absence of c-Myc. To address this question, we depleted c-Myc by AHT-inducible shRNAmediated gene silencing and supplemented the medium with Trp as before. These cells were used for infection studies with Chlamydia and the formation of progeny was analysed via Western blot (Fig. 4b, Extended data Fig. 4f). Interestingly, the pathogen failed to develop in cells with reduced levels of c-Myc even in presence of excessive exogenous Trp (Fig. 4b). Furthermore, Chlamydia was also unable to establish a secondary infection under low c-Myc expression conditions, irrespective of Trp availability (Extended data Fig. 4f). Since excess Trp cannot overcome the suppression of c-Myc, we tested if the constant expression of cMyc in a Trp free environment would be able to rescue chlamydial growth. Therefore, WIIU2OS cells were cultivated in Trp-free medium, induced with AHT, treated with IFN- $\gamma$ and infected with Chlamydia. Bacterial replication efficiency and the ability to produce infectious progeny was examined by Western blotting. Neither chlamydial development nor a secondary infection could be observed (Extended data Fig. 4g, h). In addition, c-Myc was not stabilized upon chlamydial infection in the absence of Trp (Extended data Fig. 4g), suggesting that viable bacteria and Trp are required for the stabilization of c-Myc. These data support the role of c-Myc in Trp- or indole-mediated rescue from IFN- $\gamma$-induced chlamydial persistence.

\section{L-tryptophan addition leads to activation of pGSK3ß/c-Myc axis and restores} chlamydial infection

Since we observed that Trp and c-Myc are both required for the development of Chlamydia, we next investigated the mechanism by which this amino acid regulates c-Myc level. IFN- $y$,

216 upon binding to its receptor, activates phosphatidylinositol-3-kinase ( $\mathrm{PI} K \mathrm{~K})$ and serinethreonine protein kinase (AKT) and induces the dephosphorylation and activation of glycogen 
219 of c-Myc at threonine 58, followed by its ubiquitination and proteasomal degradation ${ }^{58}$ (Fig. 4c). Both the MAPK and PI3K pathways activated during infection are critical for chlamydial development $^{59-62}$. Therefore, the phosphorylation status of AKT and GSK3ß was examined in HeLa 229 and human Fimb cells, which were treated with IFN-y and/or Trp and infected with Chlamydia. IFN-y activated the PI3 kinase pathway as evident from the phosphorylation of AKT (Fig. 4d; Extended data Fig. 4i). Despite this increase in Akt phosphorylation, IFN-y treatment strongly reduced the phosphorylation of GSK3ß and resulted in lower c-Myc levels (Fig. 4c, d; Extended data Fig. 4i). Surprisingly, the addition of Trp to HeLa or Fimb cells increased phosphorylation of GSK3ß, presumably resulting in its inactivation, and consequently led to an elevation of c-Myc levels (Fig. 4d; Extended data Fig. 4i). Taken together, these data demonstrate that Trp rescues chlamydial infection via the activation of the pGSK3ß-c-Myc axis.

IFN- $y$ induced downregulation of c-Myc has a pleiotropic effect on host metabolism

Since c-Myc is centrally involved in regulating amino acid transport ${ }^{63}$, we asked if stabilized c-Myc increases also Trp uptake. In our previous RNA-seq analysis we observed the upregulation of the L-amino acid transporter Solute Carrier Family 7 Member 5 (LAT1/SLC7A5) in cells infected with Chlamydia ${ }^{46}$. LAT1 is a system L-amino acid transporter with high affinity for branched chain and bulky amino acids, including Trp ${ }^{64}$. Furthermore, the LAT1 promoter has a binding site for c-Myc and it has been shown that overexpression of c-Myc leads to an increased expression of LAT1 ${ }^{64}$. Therefore, the protein levels of LAT1 during a chlamydial infection and upon IFN- $y$ treatment were investigated by Western blotting. Accumulation of LAT1 protein was detected in a time-dependent manner in infected HeLa 229 cells (Fig. 5a). In contrast, LAT1 protein levels were strongly reduced in IFN- $\mathrm{y}$-treated cells, irrespective of infection (Fig. 5b). Following c-Myc expression, LAT1 levels in infected cells were rescued even in the presence of IFN-Y (Fig. 5c). Interestingly, the

244 Trp-degrading enzyme IDO, which was strongly induced by IFN- $\mathrm{Y}$ treatment as expected, was only partially suppressed by c-Myc expression (Fig. 5c). 
246 To investigate how c-Myc restoration affects Trp metabolism in IFN-y treated cells, we

247 measured intracellular levels of Trp via LC-MS of WII-U2OS cells with an AHT-inducible c-

248 Myc expression. This analysis showed that Chlamydia infection increases intracellular levels

249 of Trp, while addition of IFN-y results in decreased Trp levels (Fig. 5d). Interestingly, c-Myc

250 expression in non-infected cells increased intracellular Trp to the levels of infected cells (Fig.

$2515 d$ ). However, in the presence of IFN- $\gamma$, expression of c-Myc only induced a small increase in

252 intracellular Trp levels to about the same level observed in uninfected cells (Fig. 5d),

253 indicating that $\mathrm{c}-\mathrm{Myc}$ is not sufficient to prevent the effect of IFN- $\mathrm{Y}$ on Trp degradation. To

254 also investigate whether induction of LAT1 by c-Myc affects intracellular Trp levels by

255 increasing its uptake, we also measured Trp levels in the culture medium (Fig. 5e).

256 Surprisingly, c-Myc expression had no major effect on Trp uptake in untreated and IFN-Y

257 treated cells (Fig. 5e), suggesting that restoring Trp metabolism is not the main mechanism

258 of overcoming chlamydial persistence downstream of IFN- $\gamma$ signalling.

259

260

261

262

263

264

265

266

267

268

269

270

271

272

We next performed an unbiased metabolomics analysis. WII-U2OS cells were infected with Chlamydia and either induced with $\mathrm{AHT}$, and/or treated with IFN- $\mathrm{\gamma}$, and the resulting changes in metabolite levels were analysed by LC-MS. Quality controls and data normalization were performed and a principal component analysis (PCA) demonstrated the validity of the datasets (Extended data Fig. 5a). Interestingly, hierarchical clustering analysis revealed the grouping of all conditions permissive for chlamydial development (Fig. 6a), strongly suggesting that these metabolite profiles are indicative of productive chlamydial infection. Chlamydial development is favoured in an environment with high levels of amino acids (tyrosine, histidine, alanine, homoserine, methionine, lysine, phenylalanine, threonine, asparagine, glutamate), TCA (citrate, aconitate, malate, a-ketoglutarate) and urea cycle intermediates (ornithine, citrulline) (Fig. 6a).

Pathway analysis revealed that nicotinate and nicotinamide metabolism is strongly regulated upon Chlamydia infection (Extended data Fig. 5b). Moreover, phenylalanine, tyrosine and tryptophan biosynthesis was among the significantly altered pathways with strongest impact 
273 upon infection, but also after IFN-y treatment of infected and c-Myc overexpressing infected

274 cells (Extended data Fig. 5b-d). Other modulated metabolic pathways included amino acid

275 pathways that have been shown before to be regulated upon Chlamydia infection cells ${ }^{46,65}$. In

276 addition, irrespective of IFN- $y$ treatment, arginine was one of the most depleted amino acids

277 in infected cells (Extended data Fig. 6d).

Alpha-ketoglutarate and nucleosides rescue Chlamydia from IFN-Y induced persistence

We next focused our attention on those metabolites that were induced by infection but reduced following IFN- $\mathrm{y}$ treatment and restored by c-Myc overexpression as candidates that could be causally involved in Chlamydia persistence. Chlamydia infection resulted in a significant increase in several TCA cycle (related) intermediates, including aconitate, citrate, a-ketoglutarate and glutamate (Fig. 6b; Extended data Fig. 6a). Furthermore, the amino acids aspartate, serine and glycine, which function as important precursors for TCA cycle anaplerosis and nucleotide biosynthesis (Extended data Fig. 6b), were also significantly induced by Chlamydia infection, while glutamine showed a trend towards induction that failed to reach statistical significance. In contrast, levels of arginine were strongly reduced upon infection, while intracellular levels of the urea cycle metabolites ornithine and citrulline were significantly increased (Extended data Fig. 6d).

Remarkably, treatment of infected cells with IFN-y lowered the induction of glutamate as well as aspartate, serine and glycine. Moreover, levels of most TCA cycle metabolites, in particular aconitate, citrate, $\alpha$-ketoglutarate, succinate, fumarate and malate, were also reduced upon IFN- $\gamma$ treatment, suggesting that reprogramming of host cell metabolism is a major part of the IFN-y response (Fig. 6a, b). Interestingly, re-expression of c-Myc restored levels of the TCA cycle metabolites citrate, aconitate and $\alpha$-ketoglutarate as well as the amino acids glutamine, glutamate and glycine in IFN-y treated infected cells (Fig. 6a, b;

298 Extended data Fig. 6b), suggesting that these metabolites are required for Chlamydia development. 
300 We also investigated intracellular levels of purine and pyrimidine nucleotides and

301 nucleosides as well as intermediates of nucleotide metabolism, as Chlamydia is an 302 auxotroph for nucleotides. Interestingly, IFN- $\gamma$ treatment significantly lowered the amounts of 303 ATP and CTP as well as AMP, GMP, UMP and cytidine (Extended data Fig. 6e, f; Fig. 6c). 304 Moreover, several other metabolites involved in nucleotide metabolism showed a trend 305 towards reduced abundance in IFN- $\gamma$ treated infected cells (Extended data Fig. 6e, f; Fig. 6c). This reduction in essential precursors for chlamydial DNA replication may explain why Chlamydia enter persistence in the presence of IFN-y. In addition, overexpression of c-Myc enhanced levels of nucleoside triphosphates and strongly increased intracellular levels of adenosine, uridine and cytidine in IFN-y treated cells (Fig. 6c; Extended data Fig. 6e).

Based on the observation that IFN- $y$ leads to a reduction in TCA cycle intermediates and nucleotides in host cells, we investigated whether chlamydial growth after IFN- $\gamma$ induced persistence could be overcome by the supplementation of specific metabolic precursors. We therefore treated WII-U2OS and HeLa 229 cells with IFN-ץ, followed by Chlamydia infection and addition of the cell-permeable dimethyl ester of a-ketoglutarate (DMKG) or a mixture of nucleosides (A, C, G, U). In both cell lines, the growth of Chlamydia was rescued by the addition of either DMKG or nucleosides and both conditions produced infectious progenies (Fig. 6d-f). Moreover, restoration of chlamydial development could also be achieved by the sole addition of pyrimidine nucleosides $\left(\mathrm{U}_{+} \mathrm{C}\right)$, and to a minor extend also purine nucleosides $(A+G)$ (Fig. 6f). Taken together, these results indicate that IFN- $\gamma$ broadly alters the metabolism of host cells to limit the availability of metabolic precursors for pyrimidine and purine biosynthesis to promote chlamydial persistence.

\section{Discussion}

324 Persistent and recurrent infections are an important cause of excessive inflammation and 325 tissue damage in the fallopian tube resulting in infertility and ectopic pregnancy ${ }^{66,67}$. 326 Chlamydial infection of the epithelial cells lining the genital tract leads to the secretion of 
327 cytokines, like IL-8 ${ }^{68}$ and GM-CSF ${ }^{69}$, which attract myeloid and lymphoid cells towards the 328 site of infection. This local immune response leads to chronic inflammation and may promote 329 the development of malignancy including ovarian cancer ${ }^{70}$. In addition, IFN-ץ secreted by 330 infiltrating T cells and NK cells provokes persistence of Chlamydia in epithelial cells. The current model of the immune defence elicited by IFN- $y$ in human cells centres around the induction of IDO and the consecutive degradation of $\operatorname{Trp}^{17,18}$. Chlamydia is auxotroph for Trp and enters persistence if this amino acid is degraded in the infected cell.

Our detailed molecular analysis of the IFN-y induced metabolic alterations in the host cells challenges this model and shifts the focus to the key transcription factor and proto-oncogene c-Myc as a central regulator of Chlamydia persistence. We demonstrate that IFN-y acts via the GSK3ß-STAT1 axis to deplete c-Myc levels and that constitutive expression of c-Myc is sufficient to rescue Chlamydia from persistence induced by IFN-ץ (Fig. 3). Interestingly, this effect was also reproduced in human fallopian tube organoids, a newly developed physiologically relevant model for Chlamydia infection.

The pathway governing persistence uncovered in our study was indeed dependent on the levels of Trp (Fig. 4), since Chlamydia failed to grow in the absence of this amino acid even in the presence of continuous c-Myc expression (Fig. 4; Extended date Fig. 4). Conversely, the expression of c-Myc alone without Trp provision was not sufficient to achieve normal chlamydial growth (Fig. 4; Extended data Fig. 4). Nevertheless, our results clearly show that both Trp and c-Myc are required for efficient bacterial replication. Chlamydia utilizes Trp to synthesize proteins, like the outer membrane protein MOMP, a Trp rich polypeptide. Our findings that Trp but also indole, which $C$. trachomatis can use as a substrate for Trp synthesis $^{27,55,56}$, affects the level of c-Myc was unexpected (Extended data Fig. 4). It was shown previously that glutamine deprivation lowers levels of adenosine nucleotides and suppresses c-Myc in cancer cells by a mechanism dependent on the c-Myc $3^{\prime} U_{T R}{ }^{71}$. We show here that Trp induces the phosphorylation and inactivation of GSK3ß and thereby 
data Fig. 4). Phosphorylation-dependent inactivation of GSK3ß also occurs during normal chlamydial infection and causes the accumulation of c-Myc protein, as GSK3ß leads to a destabilization of c-Myc by phosphorylation on the threonine 58 and proteasomal degradation ${ }^{58,72}$. This finding suggests that Trp activates the pGSK3ß/c-Myc axis in order to rescue $C$. trachomatis from persistence. Interestingly, c-Myc transcriptionally activates expression of the tryptophan transporter LAT-1, thereby increasing the uptake of Trp as part of a positive feed-back regulation. Moreover, Trp depletion induced by IFN-ץ signalling via STAT1 leads to loss of c-Myc expression, indicating that this amino acid functions as a central regulator of host cell metabolism and determines the decision between chlamydial development vs. the induction of persistence.

Detailed analysis of supernatants and extracts of infected cells also pointed to a broader impact of IFN- $y$ treatment on host cell metabolism. While Trp levels were significantly increased in infected cells and strongly reduced by IFN-y treatment, constitutive expression of c-Myc failed to restore Trp levels in IFN-y treated cells despite preventing Chlamydia persistence (Fig. 5d, e). This already indicated that additional metabolic pathways regulated by $\mathrm{c}-$ Myc expression may be responsible for preventing persistence induced by IFN- $\mathrm{\gamma}$. Unbiased metabolomics analysis of IFN- $y$ treated, infected and c-Myc-expressing cells and subsequent hierarchical clustering analyses revealed a grouping of all cells permissive for chlamydial replication (Fig. 6a; infected, c-Myc infected, c-Myc IFN-Y infected), suggesting that this is the metabolic profile required for permissive chlamydial replication.

Since c-Myc acts as a major metabolic regulator ${ }^{73,74}$, we systematically investigated the cMyc-dependent alterations in metabolite levels in response to bacterial infection and IFN- $\gamma$ treatment. This analysis revealed that several intermediates of the TCA cycle, including citrate, aconitate and $\alpha$-ketoglutarate, as well as glutamate, were increased upon chlamydial infection, but significantly depleted upon IFN-y-treatment and restored when c-Myc was reexpressed (Fig. 6). In addition, several nucleotides, in particular ATP and CTP were among the top metabolites upregulated upon chlamydial infection. Moreover, glutamate and alanine, 
381 all upregulated by infection, repressed in response to IFN-y treatment and restored by c-Myc 382 expression, are central metabolites for Chlamydia, since they serve as precursors for cell 383 wall biosynthesis ${ }^{75}$. Interestingly, we could observe a strong increase in the levels of citrate in c-Myc overexpressing cells (Fig. 6b). Citrate is required for the synthesis of fatty acids, which are scavenged by the bacteria from the host cell, as Chlamydia lack citrate synthase, aconitase and isocitrate dehydrogenase and thus have only an incomplete TCA cycle ${ }^{76,77}$. The finding of others that reduced uptake of glucose may play a role in IFN- $\gamma$ induced chlamydial persistence supports our model, since glycolysis fuels the TCA and nucleotide biosynthesis ${ }^{78}$.

Chlamydia-infected cells showed a drastic reduction in the levels of arginine (Extended data Fig. 6d). However, arginine levels were not restored upon c-Myc re-expression in IFN-y treated infected cells. It is possible that arginine is shuttled into the urea cycle, as we observed significantly higher levels of ornithine and citrulline (Extended data Fig. 6d), which can be used for polyamine biosynthesis, that stabilize DNA and are essential for cell proliferation $^{79}$. Further work is required to elucidate the exact role of arginine metabolism in chlamydial infection.

Here we show a central role of c-Myc in the control of the host cell metabolism during IFN- $y$ mediated innate immune defence against $C$. trachomatis infection. The effect of IFN- $y-$ mediated c-Myc downregulation included substantial remodelling of host cell metabolism, including reduced abundance of TCA cycle intermediates, which are usually replenished via c-Myc-dependent glutaminolysis and anaplerosis. Thus, the enhanced production of amino acids, nucleotides and lipids, and, additionally, TCA cycle intermediates transferred from the host cell to the bacteria ${ }^{46,80}$ may all be affected by IFN- $y$ treatment ${ }^{38}$. The central role of host TCA cycle and nucleotide biosynthesis for chlamydial development is also supported by our finding, that supplementing cell-permeable a-ketoglutarate or nucleosides can overcome the IFN-y-induced persistent state of Chlamydia (Fig. 6). It can therefore be concluded that CMyc is required for $C$. trachomatis to induce metabolic reprogramming of the host cell to 
avoid nutrient shortage during replication and prevent the induction of persistence. IFN-Y depletes the essential amino acid Trp and causes a reduction in the levels of c-Myc, which affects the metabolic state of the host cell in a way that prevents chlamydial replication and induces persistence. It is very likely, that similar mechanisms are also involved in IFN-y

412 induced persistence of other intracellular bacteria ${ }^{81}$. In addition, IFN- $\gamma$ also restricts the 413 infection of several viruses ${ }^{82,83}$. Since c-Myc activation and metabolic reprogramming of cells

414 is a prerequisite for the replication of several viruses ${ }^{84,85}$, our findings may be generally relevant for the IFN-y dependent innate immune defence against infection.

\section{Methods}

\section{Cell lines and bacteria}

Human Fimb cells (epithelial cells isolated from the fimbriae of patients undergoing hysterectomy), HeLa 229 cells (ATCC® CCL-2.1 ${ }^{\mathrm{TM}}$ ) and HeLa 229 plnducer11 shc-Myc cells were cultured in $10 \%(\mathrm{v} / \mathrm{v})$ heat inactivated FBS (Sigma-Aldrich) RPMI1640 + GlutaMAX ${ }^{\mathrm{TM}}$ medium (Gibco $\left.{ }^{T M}\right)$. WII - U2OS cells ${ }^{86}$ were maintained in high-glucose DMEM (SigmaAldrich) with $10 \%(\mathrm{v} / \mathrm{v})$ heat inactivated FBS. All cell lines were grown in a humidified atmosphere containing $5 \%(\mathrm{v} / \mathrm{v}) \mathrm{CO}_{2}$ at $37^{\circ} \mathrm{C}$. In this study Chlamydia trachomatis serovar $\mathrm{L}_{2} / 434 / \mathrm{Bu}\left(\mathrm{ATCC} \AA\right.$ VR-902B ${ }^{\mathrm{TM}}$ ) and Chlamydia trachomatis serovar D/UW-3/Cx (ATCC ${ }^{8}$

427 VR-885 ${ }^{\mathrm{TM}}$ ) were used, and cultured and purified as published previously ${ }^{87}$. In brief, 428 Chlamydia were propagated in HeLa 229 cells at a multiplicity of infection (MOI) of 1 for $48 \mathrm{~h}$. Cells were mechanically detached and lysed using glass beads ( $3 \mathrm{~mm}$, Roth). Low centrifugation supernatant $\left(10 \mathrm{~min}\right.$ at $2,000 \mathrm{~g}$ at $\left.4^{\circ} \mathrm{C}\right)$ was transferred to high speed centrifugation ( $30 \mathrm{~min}$ at $30,000 \mathrm{~g}$ at $4^{\circ} \mathrm{C}$ ) to pellet the bacteria. The pellet was washed and

432 resuspended in 1x SPG buffer (7.5\% sucrose, $0.052 \% \mathrm{KH}_{2} \mathrm{PO}_{4}, 0.122 \% \mathrm{Na}_{2} \mathrm{HPO}_{4}, 0.072 \%$

$433 \mathrm{~L}$-glutamate). Aliquots were made, stored at $-80^{\circ} \mathrm{C}$ and the bacteria were titrated to have $\mathrm{MOI}$ 
434 of 1 to be used further in the experiments. Infected cells were incubated in a humidified 435 atmosphere with $5 \%(\mathrm{v} / \mathrm{v}) \mathrm{CO}_{2}$ at $35^{\circ} \mathrm{C}$. The cell lines as well as the Chlamydia used in this 436 study were tested to be free of Mycoplasma via PCR.

Infectivity assay

For primary infection, control or IFN- $\gamma$-treated cells were infected with Chlamydia trachomatis at MOI 1 for 30-48 $\mathrm{h}$. The cells were lysed with glass beads and freshly plated cells were infected with different dilutions (referred to as secondary infection). Lysate of the secondary infection were taken to determine the infectivity via Western blotting against chlamydial HSP60.

\section{Induction and chemicals}

In order to induce c-Myc over expression in WII-U2OS cells or c-Myc knock down HeLa 229 plnducer11 shc-Myc cells, medium was changed to $10 \%(\mathrm{v} / \mathrm{v})$ heat inactivated FBS DMEM or RPMI accordingly and $100 \mathrm{ng} / \mathrm{mL}$ AHT (Anhydrotetracycline hydrochloride; Acros) was added. After $2 \mathrm{~h}$ of induction, cells were infected with Chlamydia trachomatis and incubated for further 24 to $36 \mathrm{~h}$. Interferon-gamma (Gibco ${ }^{\mathrm{TM}}$, Merck Millipore), L-tryptophan (SigmaAldrich) and Indole (Sigma-Aldrich) were added to cells as needed $2 \mathrm{~h}$ before infection.

\section{Western blot and antibodies}

For Western blot analysis, cells were directly lysed with 2x Laemmli $(10 \% 1.5 \mathrm{M}$ Tris- $\mathrm{HCl} \mathrm{pH}$ were separated with a $10 \%$ SDS-PAGE (Peqlab) and transferred onto a PVDF membrane

454 (Sigma-Aldrich) via a semi-dry blotter (Peqlab) (2 hours at $1 \mathrm{~mA}$ per $\mathrm{cm}^{2}$ ). The membrane was blocked in $5 \%(\mathrm{w} / \mathrm{v})$ non-fat dried milk powder in $1 \mathrm{x}$ Tris-buffered saline with $0.5 \%$

456 Tween20 (Sigma-Aldrich) for $1 \mathrm{~h}$ and then incubated in the appropriate primary antibody over 457 night at $4^{\circ} \mathrm{C}$; C-Myc, anti C-Myc phospho-T58 and anti C-Myc phospho-S62 were purchased from Abcam. Phospho-Akt (Ser473), Akt (pan), phospho-Erk, Erk, phospho-GSK-3ß (Ser9), 
459 GSK-3ß (3D10), phospho-Stat1 (Ser727), phospho-Stat1 (Tyr701) and Stat1 were obtained

460 from Cell Signaling. B-Actin was purchased from Sigma-Aldrich. Chlamydial HSP60 was

461 obtained from Santa Cruz and OmpA was self-made. Proteins were detected with 462 corresponding horseradish peroxidase (HRP)-conjugated secondary antibody (Santa Cruz), 463 using homemade ECL solutions and Intas Chemiluminescence Imager.

\section{Immunofluorescence analysis}

The cells were seeded on cover slips and infected with Chlamydia trachomatis serovar $\mathrm{L}_{2}$ at MOI 1 for indicated time points. Before fixation with 4\% PFA/Sucrose (Roth), the cells were washed with DPBS $\left(\right.$ Gibco $\left.^{\mathrm{TM}}\right)$. Fixed cells were permeabilized with $0.2 \%$ Triton-X-100 (Sigma-Aldrich) in 1x DPBS for 30 min, blocked with 2\% FBS in 1x DPBS for 45 min and incubated with primary antibodies for $1 \mathrm{~h}$ at room temperature. Primary antibodies were diluted in 2\% FBS in 1x DPBS; chlamydial HSP60 (Santa Cruz, 1:500) and Phalloidin

471 (Thermo Fischer). Samples were washed and incubated with fluorescence dye conjugated secondary antibodies (Dianova) for $1 \mathrm{~h}$ in the dark at room temperature. Cover slips were mounted onto microscopy slides using mowiol, slides were air dried for at least $24 \mathrm{~h}$ and examined using a LEICA DM2500 fluorescence microscope. The images were analysed with LAS AF program and Image $\mathrm{J}$ software.

\section{Metabolic profiling}

For this study, $10^{6}$ WII-U2OS cells per well were seeded in triplicates, either uninfected or infected with Chlamydia trachomatis serovar $\mathrm{L}_{2}$ for $30 \mathrm{~h}$, induced and treated with IFN- $\mathrm{\gamma}$. After the respective time, medium was collected, snap frozen in liquid nitrogen, and the cells were washed with ice cold 154mM ammonium acetate (Sigma) and snap frozen in liquid nitrogen.

481 The cells were harvested after adding $480 \mathrm{ul}$ cold $\mathrm{MeOH} / \mathrm{H}_{2} \mathrm{O}(80 / 20, \mathrm{v} / \mathrm{v})$ (Merck) to each sample containing Lamivudine (Sigma) standard $(10 \mu \mathrm{M})$. The cell suspension was collected by centrifugation and transferred to an activated (by elution of $1 \mathrm{~mL} \mathrm{CH}_{3} \mathrm{CN}$ (Merck)) and equilibrated (by elution of $1 \mathrm{~mL} \mathrm{MeOH} / \mathrm{H}_{2} \mathrm{O}(80 / 20$, v/v)) C18-E SPE-column (Phenomenex). 
485 The eluate was collected and evaporated in SpeedVac and was dissolved in $50 \mu \mathrm{L}$ $486 \mathrm{CH}_{3} \mathrm{CN} / 5 \mathrm{mM} \mathrm{NH} \mathrm{MAc}_{4}$ (25/75). Each sample was diluted 1:1 (cells) or 1:5 (medium) in $487 \mathrm{CH}_{3} \mathrm{CN} .5 \mu \mathrm{L}$ of sample was applied to HILIC column (Acclaim Mixed-Mode HILIC- 1, $3 \mu \mathrm{m}$, $2.1^{*} 150 \mathrm{~mm}$ ). Metabolites were separated at $30^{\circ} \mathrm{C}$ by LC using a DIONEX Ultimate 3000

489 UPLC system (Solvent A: 5mM NH4OAc in $\mathrm{CH}_{3} \mathrm{CN} / \mathrm{H} 2 \mathrm{O}$ (5/95), Solvent B: $5 \mathrm{mM} \mathrm{NH4OAc}$ in $490 \mathrm{CH}_{3} \mathrm{CN} / \mathrm{H} 2 \mathrm{O}$ (95/5); Gradient: linear from $100 \% \mathrm{~B}$ to $50 \% \mathrm{~B}$ in $6 \mathrm{~min}$, followed by $15 \mathrm{~min}$ 491 const. $40 \% \mathrm{~B}$, then returning to $100 \% \mathrm{~B}$ within $1 \mathrm{~min}$ ) at a flow rate of $350 \mu \mathrm{L} / \mathrm{min}$. After 492 chromatographic separation, masses $(\mathrm{m} / \mathrm{z})$ were acquired using a Q-Exactive instrument 493 (Thermo Fisher Scientific) in positive and negative ionization mode in the scan range between $69-1000 \mathrm{~m} / \mathrm{z}$ with a resolution of 70,000 . AGC target was set to $3 \times 10^{\wedge} 6$ and maximum injection time was set to $200 \mathrm{~ms}$. Sheath, auxiliary and sweep gas were set to 30, 10 and 3, respectively, and spray voltage was fixed to $3.6 \mathrm{kV}$. S-lens RF level was set to 55.0 , the capillary and the Aux gas heater were heated to $320^{\circ} \mathrm{C}$ and $120^{\circ} \mathrm{C}$, respectively. Peak determination and semi-quantitation were performed using TraceFinder $^{\mathrm{TM}}$ Software. Obtained signal intensities were normalized to the internal standard (lamivudine) and a cell number, which was determined by crystal violet staining. In brief, for the crystal violet staining cells were fixed with $4 \%$ PFA/Sucrose (Roth), stained with $0.1 \%$ crystal violet (Merck) dissolved in $20 \%$ ethanol (Roth), washed with water, dried overnight and the absorbance was measured at $550 \mathrm{~nm}$. The pellet of the cell samples was dried, resuspended in $0.2 \mathrm{M}$ sodium hydroxide (Roth), cooked for $20 \mathrm{~min}$ at $95^{\circ} \mathrm{C}$ and absorbance was measured at 550nm. Statistical analysis was performed using Prism GraphPad. Hierarchical clustering, 506 principal component analyses (PCA) and pathway analyses were done and resulting plots were generated by MetaboAnalyst $4.0^{88}$.

\section{Generation of human fallopian tube organoids}

509 Generation of human organoids was adapted from $^{89}$. Fallopian tube tissue was obtained 510 from patients who underwent hysterectomy. The tissue was prepared and processed within 2 511 h. Briefly, tissue samples were washed with DPBS (Gibco) and placed into a sterile Petri dish 
512 (Corning) where they were cut into small pieces. Then, on the top of the minced tissue, a 513 glass slide (VWR) was placed and strongly pressed down to obtain smaller pieces. The cells 514 were washed with DPBS, placed into a $15 \mathrm{~mL}$ falcon tube and centrifuged at $1,000 \mathrm{~g}$ for 10 $515 \mathrm{~min}$. The supernatant was removed, the pellet was resuspended in Matrigel (Corning) and 516 plated in $50 \mu \mathrm{L}$ drop in wells in a 24 -well plate. The plate was carefully transferred to $37^{\circ} \mathrm{C}$ 517 incubator to allow the Matrigel to get solidified for $20 \mathrm{~min}$ following the addition $500 \mu \mathrm{L} /$ well of 518 pre-warmed media (DMEM advanced (Sigma), Wnt (25\%), R-Spondin (25\%), Noggin (10\%), 519 B27 (2\%; Thermo Scientific), Nicotinamide (1 mM; Sigma), human EGF (50 ng/mL; Thermo 520 Scientific), FGF (100 ng/mL; Thermo Scientific), TGF-ß inhibitor (0.5 mM; Tocris), Rock 521 inhibitor (10 mM; Abmole Bioscience).

\section{Splitting organoids}

Approximately in 7 days, the Matrigel drop was carefully resuspended in cold DMEM medium and centrifuged at $1,000 \mathrm{~g}$ at $4^{\circ} \mathrm{C}$ for $5 \mathrm{~min}$. The supernatant was discarded and $50 \mu \mathrm{L}$ Matrigel was added and further processed as explained above.

\section{Infectivity assay in organoids}

527 For infection with $C$. trachomatis mature organoids were released from a confluent Matrigel 528 drop by resuspending it with ice-cold DPBS (Gibco). The suspension was collected in a lowbinding Eppendorf tube and infected with $C$. trachomatis $\mathrm{L}_{2}\left(5 \times 10^{5} \mathrm{IFU}\right)$. The suspension was mixed and placed on ice for 30 min following centrifugation. $50 \mu \mathrm{L}$ of Matrigel was added to each tube and seeded into a 24-well plate (Corning) with following 20 min incubation at

$53237^{\circ} \mathrm{C}$ to allow the Matrigel drop solidify. Six days post infection the organoids were fixed with 4\% PFA and used for immunostaining. In addition, infected organoids were lysed with glass beads and different dilutions were used to infect freshly plated HeLa 229 cells to analyse the infectivity of the progeny. 
538 We thank Dr. Jörg Wischhusen for providing the human Fimb cells and Dr. Francesca Dejure

539 for suppling us with WII-U2OS cells. We thank Dr. Andreas Demuth for critically reading the

540 manuscript. This work was supported by the Deutsche Forschungsgemeinschaft (DFG)

541 priority program GRK2157 "3D Tissue Models for Studying Microbial Infections by Human

542 Pathogens" and the European Research Council (grant no. ERC-2018-ADG/NCI-CAD) to

543 T.R.

\section{Author contribution}

545 N.V., K.R. and T.R. conceived and designed the study; N.V., K.R., N.K. and S.J.R. 546 performed the experiments; S.J.R, N.V, L.S., W.S. and A.S. performed the mass spec 547 analysis. N.V., T.R and K.R. wrote the manuscript, further edited by S.J.R., L.S., and A.S.; 548 T.R, financially supported the project. K.R. supervised the work.

\section{Declaration of interests}

The authors declare no competing interests.

1 Fenwick, A. The global burden of neglected tropical diseases. Public Health 126, 233-236, doi:https://doi.org/10.1016/i.puhe.2011.11.015 (2012).

2 Newman, L. et al. Global Estimates of the Prevalence and Incidence of Four Curable Sexually Transmitted Infections in 2012 Based on Systematic Review and Global Reporting. PLOS ONE 10, e0143304, doi:10.1371/journal.pone.0143304 (2015).

3 Svenstrup, H. F. et al. Mycoplasma genitalium, Chlamydia trachomatis, and tubal factor infertility-a prospective study. Fertility and Sterility 90, 513-520, doi:https://doi.org/10.1016/i.fertnstert.2006.12.056 (2008).

4 Malik, A., Jain, S., Rizvi, M., Shukla, I. \& Hakim, S. Chlamydia trachomatis infection in women with secondary infertility. Fertility and Sterility 91, 91-95, doi:https://doi.org/10.1016/i.fertnstert.2007.05.070 (2009).

5 Ho, J. L. et al. Neutrophils from human immunodeficiency virus (HIV)seronegative donors induce HIV replication from HIV-infected patients' mononuclear cells and cell lines: an in vitro model of HIV transmission facilitated by Chlamydia trachomatis. The Journal of Experimental Medicine 181, 1493-1505 (1995).

6 Galvin, S. R. \& Cohen, M. S. The role of sexually transmitted diseases in HIV transmission. Nature Reviews Microbiology 2, 33, doi:10.1038/nrmicro794 (2004). 7 Hare, M. J., TAYLOR-ROBINSON, D. \& COOPER, P. Evidence for an association between Chlamydia trachomatis and cervical intraepithelial neoplasia. BJOG: An International Journal of Obstetrics \& Gynaecology 89, 489-492, doi:doi:10.1111/j.1471-0528.1982.tb03643.x (1982). 
8 Koskela, P. et al. Chlamydia trachomatis infection as a risk factor for invasive cervical cancer. International Journal of Cancer 85, 35-39, doi:doi:10.1002/(SICI)1097-0215(20000101)85:1<35::AID-IJC6>3.0.CO;2-A (2000). 9 Smith, J. S. et al. Chlamydia trachomatis and invasive cervical cancer: A pooled analysis of the IARC multicentric case-control study. International Journal of Cancer 111, 431-439, doi:doi:10.1002/ijc.20257 (2004).

10 Gagnaire, A., Nadel, B., Raoult, D., Neefjes, J. \& Gorvel, J.-P. Collateral damage: insights into bacterial mechanisms that predispose host cells to cancer. Nature Reviews Microbiology 15, 109, doi:10.1038/nrmicro.2016.171 (2017). 11 Wang, Y. et al. Development of a Transformation System for Chlamydia trachomatis: Restoration of Glycogen Biosynthesis by Acquisition of a Plasmid Shuttle Vector. PLoS Pathogens 7, e1002258, doi:10.1371/journal.ppat.1002258 (2011). 12 Gaylord, W. H. INTRACELLULAR FORMS OF MENINGOPNEUMONITIS VIRUS. The Journal of Experimental Medicine 100, 575-580, doi:10.1084/jem.100.6.575 (1954). 13 Hackstadt, T., Fischer, E. R., Scidmore, M. A., Rockey, D. D. \& Heinzen, R. A. Origins and functions of the chlamydial inclusion. Trends in Microbiology 5, 288-293, doi:https://doi.org/10.1016/S0966-842X(97)01061-5 (1997). 14 AbdelRahman, Y. M. \& Belland, R. J. The chlamydial developmental cycle. FEMS Microbiology Reviews 29, 949-959, doi:10.1016/j.femsre.2005.03.002 (2005). 15 Hybiske, K. \& Stephens, R. S. Mechanisms of host cell exit by the intracellular bacterium Chlamydia. Proceedings of the National Academy of Sciences of the United States of America 104, 11430-11435, doi:10.1073/pnas.0703218104 (2007).

16 Suchland, R. J., Dimond, Z. E., Putman, T. E. \& Rockey, D. D. Demonstration of Persistent Infections and Genome Stability by Whole-Genome Sequencing of RepeatPositive, Same-Serovar Chlamydia trachomatis Collected From the Female Genital Tract. J Infect Dis 215, 1657-1665, doi:10.1093/infdis/jix155 (2017). 17 Wyrick, P. B. Chlamydia trachomatis Persistence in Vitro-An Overview. The Journal of infectious diseases 201, S88-S95, doi:10.1086/652394 (2010).

18 Beatty, W. L., Belanger, T. A., Desai, A. A., Morrison, R. P. \& Byrne, G. I. Tryptophan depletion as a mechanism of gamma interferon-mediated chlamydial persistence. Infection and Immunity 62, 3705-3711 (1994).

19 Tamura, A. \& Manire, G. P. Effect of Penicillin on the Multiplication of Meningopneumonitis Organisms (Chlamydia psittaci). Journal of Bacteriology 96, 875-880 (1968).

20 Raulston, J. E. Response of Chlamydia trachomatis serovar E to iron restriction in vitro and evidence for iron-regulated chlamydial proteins. Infection and Immunity 65, 4539-4547 (1997).

21 Allan, I. \& Pearce, J. H. Differential Amino Acid Utilization by Chlamydia psittaci (Strain Guinea Pig Inclusion Conjunctivitis) and its Regulatory Effect on Chlamydial Growth. Microbiology 129, 1991-2000, doi:doi:10.1099/00221287-129-71991 (1983).

22 MacMicking, J. D. Interferon-inducible effector mechanisms in cellautonomous immunity. Nat Rev Immunol 12, 367-382, doi:10.1038/nri3210 (2012). 23 Nelson, D. E. et al. Chlamydial IFN-gamma immune evasion is linked to host infection tropism. Proc Natl Acad Sci U S A 102, 10658-10663, doi:10.1073/pnas.0504198102 (2005).

24 Taylor, M. W. \& Feng, G. S. Relationship between interferon-gamma, indoleamine 2,3-dioxygenase, and tryptophan catabolism. Faseb j 5, 2516-2522 (1991).

25 Ostergaard, O. et al. Quantitative Protein Profiling of Chlamydia trachomatis Growth Forms Reveals Defense Strategies Against Tryptophan Starvation. Mol Cell Proteomics 15, 3540-3550, doi:10.1074/mcp.M116.061986 (2016). 
26 Wyrick, P. B. Chlamydia trachomatis persistence in vitro: an overview. J Infect Dis 201 Suppl 2, S88-95, doi:10.1086/652394 (2010).

27 Byrne, G. I., Lehmann, L. K. \& Landry, G. J. Induction of tryptophan catabolism is the mechanism for gamma-interferon-mediated inhibition of intracellular Chlamydia psittaci replication in T24 cells. Infection and Immunity 53, 347-351 (1986).

28 Ramana, C. V. et al. Regulation of c-myc expression by IFN- $\gamma$ through Stat1dependent and -independent pathways. The EMBO Journal 19, 263-272, doi:10.1093/emboj/19.2.263 (2000).

29 Krause, C. D., He, W., Kotenko, S. \& Pestka, S. Modulation of the activation of Stat1 by the interferon- $\gamma$ receptor complex. Cell Research 16, 113, doi:10.1038/sj.cr.7310015 (2006).

$30 \mathrm{Hu}, \mathrm{X}$. \& Ivashkiv, L. B. Cross-regulation of Signaling Pathways by Interferon- $\gamma$ : Implications for Immune Responses and Autoimmune Diseases. Immunity 31, 539550, doi:https://doi.org/10.1016/j.immuni.2009.09.002 (2009).

31 Ramana, C. V. et al. Regulation of c-myc expression by IFN-gamma through Stat1-dependent and -independent pathways. EMBO J 19, 263-272, doi:10.1093/emboj/19.2.263 (2000). 32 Asao, H. \& Fu, X. Y. Interferon-gamma has dual potentials in in hibiting or promoting cell proliferation. J Biol Chem 275, 867-874, doi:10.1074/jbc.275.2.867 (2000).

33 Schlee, M. et al. c-MYC activation impairs the NF-KB and the interferon response: Implications for the pathogenesis of Burkitt's lymphoma. International Journal of Cancer 120, 1387-1395, doi:doi:10.1002/ijc.22372 (2007).

34 Duesberg, P. H. \& Vogt, P. K. Avian acute leukemia viruses MC29 and MH2 share specific RNA sequences: Evidence for a second class of transforming genes. Proceedings of the National Academy of Sciences of the United States of America 76, 1633-1637 (1979).

35 Coffin, J. M. et al. Proposal for Naming Host Cell-Derived Inserts in Retrovirus Genomes. Journal of Virology 40, 953-957 (1981).

36 Battey, J. et al. The human c-myc oncogene: Structural consequences of translocation into the igh locus in Burkitt lymphoma. Cell 34, 779-787, doi:https://doi.org/10.1016/0092-8674(83)90534-2 (1983).

37 Dang, C. V. c-Myc target genes involved in cell growth, apoptosis, and metabolism. Mol Cell Biol 19, 1-11, doi:10.1128/mcb.19.1.1 (1999).

38 Kress, T. R., Sabò, A. \& Amati, B. MYC: connecting selective transcriptional control to global RNA production. Nature Reviews Cancer 15, 593, doi:10.1038/nrc3984 (2015).

39 Liu, Y. C. et al. Global regulation of nucleotide biosynthetic genes by c-Myc. PLoS One 3, e2722, doi:10.1371/journal. pone.0002722 (2008).

40 Mannava, S. et al. Direct role of nucleotide metabolism in C-MYC-dependent proliferation of melanoma cells. Cell Cycle 7, 2392-2400, doi:10.4161/cc.6390 (2008). 41 Cunningham, J. T., Moreno, M. V., Lodi, A., Ronen, S. M. \& Ruggero, D. Protein and nucleotide biosynthesis are coupled by a single rate-limiting enzyme, PRPS2, to drive cancer. Cell 157, 1088-1103, doi:10.1016/j.cell.2014.03.052 (2014). 42 Evans, D. R. \& Guy, H. I. Mammalian pyrimidine biosynthesis: fresh insights into an ancient pathway. J Biol Chem 279, 33035-33038, doi:10.1074/jbc.R400007200 (2004).

43 Makinoshima, H. et al. Epidermal growth factor receptor (EGFR) signaling regulates global metabolic pathways in EGFR-mutated lung adenocarcinoma. J Biol Chem 289, 20813-20823, doi:10.1074/jbc.M114.575464 (2014). 
44 Gordan, J. D., Thompson, C. B. \& Simon, M. C. HIF and c-Myc: sibling rivals for control of cancer cell metabolism and proliferation. Cancer Cell 12, 108-113, doi:10.1016/j.ccr.2007.07.006 (2007).

45 Khan, S., Abdelrahim, M., Samudio, I. \& Safe, S. Estrogen receptor/Sp1 complexes are required for induction of cad gene expression by 17 beta-estradiol in breast cancer cells. Endocrinology 144, 2325-2335, doi:10.1210/en.2002-0149 (2003).

46 Rajeeve, K. et al. Reprogramming of host glutamine metabolism during Chlamydia trachomatis infection and its key role in peptidoglycan synthesis. Nat Microbiol, doi:10.1038/s41564-020-0762-5 (2020).

47 Tipples, G. \& McClarty, G. The obligate intracellular bacterium Chlamydia trachomatis is auxotrophic for three of the four ribonucleoside triphosphates. Mol Microbiol 8, 1105-1114, doi:10.1111/j.1365-2958.1993.tb01655.x (1993).

48 McClarty, G. \& Tipples, G. In situ studies on incorporation of nucleic acid precursors into Chlamydia trachomatis DNA. J Bacteriol 173, 4922-4931, doi:10.1128/jb.173.16.4922-4931.1991 (1991).

49 Aiyar, A. et al. Influence of the tryptophan-indole-IFNgamma axis on human genital Chlamydia trachomatis infection: role of vaginal co-infections. Front Cell Infect Microbiol 4, 72, doi:10.3389/fcimb.2014.00072 (2014).

50 Panzetta, M. E., Valdivia, R. H. \& Saka, H. A. Chlamydia Persistence: A Survival Strategy to Evade Antimicrobial Effects in-vitro and in-vivo. Front Microbiol 9, 3101, doi:10.3389/fmicb.2018.03101 (2018).

51 Raulston, J. E. Response of Chlamydia trachomatis serovar $E$ to iron restriction in vitro and evidence for iron-regulated chlamydial proteins. Infect Immun 65, 4539-4547 (1997).

52 Allan, I. \& Pearce, J. H. Amino acid requirements of strains of Chlamydia trachomatis and C. psittaci growing in McCoy cells: relationship with clinical syndrome and host origin. J Gen Microbio/ 129, 2001-2007, doi:10.1099/00221287129-7-2001 (1983).

53 Vervoorts, J., Luscher-Firzlaff, J. \& Luscher, B. The ins and outs of MYC regulation by posttranslational mechanisms. J Biol Chem 281, 34725-34729, doi:10.1074/jbc.R600017200 (2006).

54 Welcker, M. et al. The Fbw7 tumor suppressor regulates glycogen synthase kinase 3 phosphorylation-dependent c-Myc protein degradation. Proc Natl Acad Sci U S A 101, 9085-9090, doi:10.1073/pnas.0402770101 (2004).

$55 \quad$ Kari, L. et al. Generation of targeted Chlamydia trachomatis null mutants. Proceedings of the National Academy of Sciences of the United States of America 108, 7189-7193, doi:10.1073/pnas.1102229108 (2011).

56 Østergaard, O. et al. Quantitative Protein Profiling of Chlamydia trachomatis Growth Forms Reveals Defense Strategies Against Tryptophan Starvation. Molecular \& Cellular Proteomics : MCP 15, 3540-3550, doi:10.1074/mcp.M116.061986 (2016). 57 Nguyen, H., Ramana, C. V., Bayes, J. \& Stark, G. R. Roles of phosphatidylinositol 3-kinase in interferon-gamma-dependent phosphorylation of STAT1 on serine 727 and activation of gene expression. J Biol Chem 276, 3336133368, doi:10.1074/jbc.M105070200 (2001).

58 Albert, T., Urlbauer, B., Kohlhuber, F., Hammersen, B. \& Eick, D. Ongoing mutations in the N-terminal domain of C-Myc affect transactivation in Burkitt's lymphoma cell lines. Oncogene 9, 759-763 (1994).

59 Capmany, A., Gambarte Tudela, J., Alonso Bivou, M. \& Damiani, M. T. Akt/AS160 Signaling Pathway Inhibition Impairs Infection by Decreasing Rab14Controlled Sphingolipids Delivery to Chlamydial Inclusions. Front Microbio/ 10, 666, doi:10.3389/fmicb.2019.00666 (2019). 
60 Patel, A. L. et al. Activation of epidermal growth factor receptor is required for Chlamydia trachomatis development. BMC Microbiol 14, 277, doi:10.1186/s12866-014-0277-4 (2014).

61 Subbarayal, P. et al. EphrinA2 receptor (EphA2) is an invasion and intracellular signaling receptor for Chlamydia trachomatis. PLoS Pathog 11, e1004846, doi:10.1371/journal.ppat.1004846 (2015).

62 Siegl, C., Prusty, B. K., Karunakaran, K., Wischhusen, J. \& Rudel, T. Tumor suppressor p53 alters host cell metabolism to limit Chlamydia trachomatis infection. Cell Rep 9, 918-929, doi:10.1016/j.celrep.2014.10.004 (2014).

63 Dong, Y., Tu, R., Liu, H. \& Qing, G. Regulation of cancer cell metabolism: oncogenic MYC in the driver's seat. Signal Transduct Target Ther 5, 124, doi:10.1038/s41392-020-00235-2 (2020).

64 Bhutia, Y. D., Babu, E. \& Ganapathy, V. Interferon- $\gamma$ induces a tryptophanselective amino acid transporter in human colonic epithelial cells and mouse dendritic cells. Biochimica et Biophysica Acta (BBA) - Biomembranes 1848, 453-462, doi:https://doi.org/10.1016/i.bbamem.2014.10.021 (2015).

65 Mehlitz, A. et al. Metabolic adaptation of Chlamydia trachomatis to mammalian host cells. Mol Microbiol 103, 1004-1019, doi:10.1111/mmi.13603 (2017).

66 Nagarajan, U. M., Sikes, J. D., Yeruva, L. \& Prantner, D. Significant role of IL-1 signaling, but limited role of inflammasome activation, in oviduct pathology during Chlamydia muridarum genital infection. J Immunol 188, 2866-2875, doi:10.4049/jimmunol.1103461 (2012).

67 Darville, T. et al. Toll-like receptor-2, but not Toll-like receptor-4, is essential for development of oviduct pathology in chlamydial genital tract infection. J Immunol 171, 6187-6197, doi:10.4049/jimmunol.171.11.6187 (2003).

68 Buchholz, K. R. \& Stephens, R. S. The cytosolic pattern recognition receptor NOD1 induces inflammatory interleukin-8 during Chlamydia trachomatis infection. Infect Immun 76, 3150-3155, doi:10.1128/IAI.00104-08 (2008).

69 Lehr, S., Vier, J., Hacker, G. \& Kirschnek, S. Activation of neutrophils by Chlamydia trachomatis-infected epithelial cells is modulated by the chlamydial plasmid. Microbes Infect 20, 284-292, doi:10.1016/j.micinf.2018.02.007 (2018). 70 Shan, W. \& Liu, J. Inflammation: a hidden path to breaking the spell of ovarian cancer. Cell Cycle 8, 3107-3111, doi:10.4161/cc.8.19.9590 (2009).

71 Dejure, F. R. et al. The MYC mRNA 3'-UTR couples RNA polymerase II function to glutamine and ribonucleotide levels. EMBO J 36, 1854-1868, doi:10.15252/embj.201796662 (2017).

72 Al-Zeer, M. A. et al. Chlamydia trachomatis Prevents Apoptosis Via Activation of PDPK1-MYC and Enhanced Mitochondrial Binding of Hexokinase II. EBioMedicine 23, 100-110, doi:10.1016/j.ebiom.2017.08.005 (2017).

73 Dejure, F. R. \& Eilers, M. MYC and tumor metabolism: chicken and egg. EMBO J 36, 3409-3420, doi:10.15252/embj.201796438 (2017).

74 Stine, Z. E., Walton, Z. E., Altman, B. J., Hsieh, A. L. \& Dang, C. V. MYC, Metabolism, and Cancer. Cancer Discov 5, 1024-1039, doi:10.1158/2159-8290.CD-150507 (2015).

75 Otten, C., Brilli, M., Vollmer, W., Viollier, P. H. \& Salje, J. Peptidoglycan in obligate intracellular bacteria. Mol Microbiol 107, 142-163, doi:10.1111/mmi.13880 (2018).

76 Yao, J., Dodson, V. J., Frank, M. W. \& Rock, C. O. Chlamydia trachomatis Scavenges Host Fatty Acids for Phospholipid Synthesis via an Acyl-Acyl Carrier Protein Synthetase. J Biol Chem 290, 22163-22173, doi:10.1074/jbc.M115.671008 (2015). 
77 Yao, J. et al. Type II Fatty Acid Synthesis Is Essential for the Replication of Chlamydia trachomatis. The Journal of Biological Chemistry 289, 22365-22376, doi:10.1074/jbc.M114.584185 (2014).

78 Shima, K. et al. Interferon-gamma interferes with host cell metabolism during intracellular Chlamydia trachomatis infection. Cytokine 112, 95-101, doi:10.1016/j.cyto.2018.05.039 (2018).

79 van Dam, L., Korolev, N. \& Nordenskiold, L. Polyamine-nucleic acid interactions and the effects on structure in oriented DNA fibers. Nucleic Acids Res 30, 419-428, doi:10.1093/nar/30.2.419 (2002).

80 Adrian, M. et al. Metabolic adaptation of Chlamydia trachomatis to mammalian host cells. Molecular Microbiology 103, 1004-1019, doi:doi:10.1111/mmi.13603 (2017).

81 Ganesan, S. \& Roy, C. R. Host cell depletion of tryptophan by IFNgammainduced Indoleamine 2,3-dioxygenase 1 (IDO1) inhibits lysosomal replication of Coxiella burnetii. PLoS Pathog 15, e1007955, doi:10.1371/journal.ppat.1007955 (2019).

82 Weizman, O. E. et al. ILC1 Confer Early Host Protection at Initial Sites of Viral Infection. Cell 171, 795-808 e712, doi:10.1016/j.cell.2017.09.052 (2017).

83 Karupiah, G. et al. Inhibition of viral replication by interferon-gamma-induced nitric oxide synthase. Science 261, 1445-1448, doi:10.1126/science.7690156 (1993). 84 Thai, M. et al. Adenovirus E4ORF1-induced MYC activation promotes host cell anabolic glucose metabolism and virus replication. Cell Metab 19, 694-701, doi:10.1016/j.cmet.2014.03.009 (2014). 85 Thai, M. et al. MYC-induced reprogramming of glutamine catabolism supports optimal virus replication. Nat Commun 6, 8873, doi:10.1038/ncomms9873 (2015).

86 Lorenzin, F. et al. Different promoter affinities account for specificity in MYCdependent gene regulation. elife 5, e15161, doi:10.7554/eLife.15161 (2016).

87 Paland, N. et al. Reduced display of tumor necrosis factor receptor I at the host cell surface supports infection with Chlamydia trachomatis. J Biol Chem 283, 6438-6448, doi:10.1074/jbc.M708422200 (2008).

88 Chong, J., Wishart, D. S. \& Xia, J. Using MetaboAnalyst 4.0 for Comprehensive and Integrative Metabolomics Data Analysis. Curr Protoc Bioinformatics 68, e86, doi:10.1002/cpbi.86 (2019).

89 Kessler, M. et al. Chronic Chlamydia infection in human organoids increases stemness and promotes age-dependent CpG methylation. Nat Commun 10, 1194, doi:10.1038/s41467-019-09144-7 (2019).

\section{Figure legends}

\section{Figure 1: IFN-y induces depletion of c-Myc and impairs chlamydial growth.}

a. HeLa 229 cells with an AHT-inducible expression of shc-Myc were infected with Chlamydia at MOI 1. The infected cells were either left untreated or treated with $1 \mathrm{ng} / \mathrm{mL}$ AHT to deplete c-Myc $8 \mathrm{~h}$ before infection. After $24 \mathrm{~h}$ of infection, AHT was removed to release c-Myc expression and the restoration of inclusion formation was tested. Cells were 
825 either fixed with 4\% PFA after 36 hpi and immunostained for Ctr (cHSP60: green) and DNA

826 (DAPI: blue) or lysed and analysed by Western blot in order to analyse the rescue.

827 Additionally, infected cells were lysed to infect freshly plated HeLa 229 shc-Myc cells at 24

828 hpi and analysed via Western blot to investigate the formation of infectious progenies. The

829 panel shows representative images $(n=3)$. cHSP60 indicates Chlamydia infection and B-Actin

830 serve as the loading control $(n=3)$. b. HeLa 229 cells were either left untreated or were pre-

831 treated for $2 \mathrm{~h}$ with $10 \mathrm{ng} / \mathrm{mL} \mathrm{IFN}-\mathrm{y}$ or 1 unit of penicillin, infected with $\mathrm{Ctr}$ at $\mathrm{MOI} 1$ for

832 different time intervals and lysed for Western blot analysis. Bacterial load (cHSP60) and c-

833 Myc levels were determined and $\beta$-Actin served as loading control $(n=3)$. c. HeLa 229 cells

834 were either left untreated or were pre-treated for $2 \mathrm{~h}$ with $10 \mathrm{ng} / \mathrm{mL}$ of IFN- $\mathrm{y}$ and infected with

835 Chlamydia (Ctr) at MOI 1 and lysed at $30 \mathrm{~h}$ to perform Western blot analysis. Phosphorylated

836 form of c-Myc at serine 62 (pc-Myc (Ser62)) and threonine 58 (pc-Myc (Thr58)), c-Myc and

837 Chlamydia (cHSP60) were detected and quantified (Fc) $(n=3)$. d. Cartoon depicting IFN-v

838 signalling. IFN- $\gamma$ binds to the IFN- $\gamma$ receptor that results in the phosphorylation of STAT1.

839 pSTAT1 binds to the GAS sequence and blocks c-Myc transcription. IFN- $y$ can also induce

840 indoleamine-2,3-dioxygenase (IDO) and thereby the degradation of L-tryptophan. e. HeLa

841229 cells were either left untreated or treated with $10 \mathrm{ng} / \mathrm{mL}$ of IFN- $\gamma$ and infected with Ctr.

842 The cells were lysed and relative mRNA levels of c-Myc were determined by qPCR. GAPDH

843 was used for normalization $(n=3) .{ }^{* *}$ indicates $p$ value $<0.001$ and $n s$ indicates non-

844 significant. f. HeLa 229 cells were either left untreated or were pre-treated for 2 h with 10

$845 \mathrm{ng} / \mathrm{mL}$ of IFN-y and infected with Chlamydia (Ctr) at MOI 1 and lysed at $30 \mathrm{hpi}$ to study

846 STAT1 signalling after Ctr infection $(\mathrm{n}=3)$. g. HeLa 229 cells were transfected with siRNA

847 against STAT1 (+) or control (-) for $48 \mathrm{~h}$ and then infected with Ctr for $24 \mathrm{~h}$. The cells were

848 lysed and analysed by Western blot to investigate the STAT1 signalling and infectivity of Ctr $849(n=3)$.

850 For all blots shown in figure 1, Chlamydia load (cHSP60) and the respective host cell protein

851 levels were quantified by normalization to B-Actin and indicated as fold change (Fc). 


\section{Figure 2: IFN-Y induces depletion of $\mathrm{c}-\mathrm{Myc}$ and impairs chlamydial growth in human}

\section{3 fallopian tube organoids.}

854 a. Cartoon depicting how the infectivity assay was performed in human fallopian tube 855 organoids. Organoids (see Methods) were infected with Ctr and treated with or without IFN-y 856 for 6 days and lysed using glass beads. Dilutions of the supernatants were used to infect 857 freshly plated HeLa 229 cells. b. Human fallopian tube organoids were infected with Ctr and 858 treated with or without IFN-y for 6 days. The organoids were fixed with $4 \%$ PFA and 859 immunostained for DNA (DAPI: blue), Ctr (cHSP60: green), Cytokeratin (magenta), 860 Phalloidin (light blue). The panel shows representative images from organoids derived from

861 five patients. c. The infected organoids from (b) were lysed using glass beads, and dilutions 862 of the supernatant were used to infect freshly plated HeLa 229 cells. The number of 863 inclusions as shown in (d) were counted from 5 different patients and mean \pm SD depicted in 864 the graph $(n=5) .{ }^{* *}$ indicates $p$ value $<0.01$. e. Human fallopian tube organoids were infected 865 with Ctr and treated with or without IFN-y for 6 days. The organoids were lysed in $2 x$ 866 Laemmeli buffer and analysed by Western blotting. Chlamydia load (cHSP60) and STAT1 867 protein levels were quantified by normalization to B-Actin and indicated as fold change (Fc).

Figure 3: Expression of c-Myc rescues Chlamydia from persistence.

a. WII-U2OS cells were induced with $100 \mathrm{ng} / \mathrm{mL}$ AHT for $2 \mathrm{~h}$. Cells were left untreated or

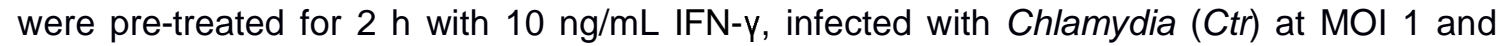
lysed at 30 hpi for Western blot analysis. b. The infected cells from (a) were lysed to infect freshly plated HeLa 229 cells. The numbers of inclusions were counted from the different conditions shown in (a). The mean \pm SD are shown in the graph. ${ }^{* * *} p<0.001$, ns $=$ not significant. c. For the same culture conditions as in (a), an infectivity assay was performed. The HeLa 229 cells were fixed with 4\% PFA after 30 hpi and immunostained for Ctr (cHSP60: green) and actin (Phalloidin: blue) in order to analyse the rescue. d. From the experiment shown in (e) the infected/ IFN-y treated organoids were lysed with glass beads and different dilutions of the supernatant was used to infect freshly plated HeLa 229 cells. 
879 The number of inclusions were counted from three different experiments and mean \pm SD are

880 shown in the graph. ${ }^{* *}$ indicates a $p$ value $<0.01$. e. The organoids from (Extended data

881 Fig. 3b) were infected with Ctr for 6 days with and without IFN- $\gamma$, were lysed using glass

882 beads, and dilutions of the supernatant were used to infect freshly plated HeLa 229 cells for

883 an infectivity assay. Cells were fixed with 4\% PFA after $30 \mathrm{hpi}$ and immunostained for Ctr

884 (cHSP60: green) and actin (Phalloidin: red) in order to analyse the rescue.

Figure 4: L-tryptophan activates the pGSK3ß-c-Myc axis and rescues chlamydial infection.

a. HeLa 229 cells were treated with IFN-y and different doses of L-tryptophan (W) (10-100 $\mathrm{ng} / \mathrm{mL})$. After $30 \mathrm{~h}$ the cells were lysed and used for Western blot analysis $(n=3)$. b. HeLa 229 cells with an AHT-inducible expression of shc-Myc were either left uninfected or infected with Chlamydia at MOI 1 for $30 \mathrm{~h}$. The infected cells were either left untreated or treated with L-tryptophan and $1 \mathrm{ng} / \mathrm{mL}$ AHT to deplete c-Myc. The cells were further analysed via Western blotting $(n=3)$. c. Cartoon showing how IFN-y signals lead to c-Myc depletion. Left side, active Phosphatidylinositol-3-kinase (PI3K) with inactive glycogen synthase kinase-3 (GSK3ß) leading to c-Myc stabilization. Right side, IFN- $y$ binding to its receptor, activates $\mathrm{PI} 3 \mathrm{~K}$ and serine-threonine protein kinase (AKT) and induces the dephosphorylation and activation of GSK3ß, leading to c-Myc depletion. Chlamydia infection activates the PI3K- and MEK/ERK-pathway. d. HeLa 229 cells were either left uninfected or infected with Ctr and treated with IFN- $\gamma$ with or without L-tryptophan (W). The cells were analysed via Western blotting after 30 hpi $(n=3)$. cHSP60 indicates Chlamydia infection and B-Actin serve as the loading control.

Figure 5: Influence of stabilized c-Myc on Trp uptake and metabolites.

902

903

904

905

a. HeLa 229 cells were infected with Chlamydia (Ctr) for various time points. The cells were analysed via Western blotting for the levels of LAT1 $(n=3)$. b. HeLa 229 cells were either left untreated or were pre-treated for $2 \mathrm{~h}$ with $10 \mathrm{ng} / \mathrm{mL}$ of IFN- $\mathrm{y}$, infected with Ctr at MOI 1 and lysed at 24 hpi to examine LAT1 regulation via Western blotting. c. WII-U2OS cells were 
906 induced with $100 \mathrm{ng} / \mathrm{mL}$ AHT for $2 \mathrm{~h}$. Cells were left untreated or were pre-treated for $2 \mathrm{~h}$

907 with $10 \mathrm{ng} / \mathrm{mL}$ IFN- $\mathrm{\gamma}$, infected with Chlamydia (Ctr) at MOI 1 and lysed at $24 \mathrm{hpi}$ for Western

908 blot analysis $(n=3)$. d. WII-U2OS cells were either left uninfected or infected with Ctr at MOI 1

909 for $30 \mathrm{~h}$. The infected cells were either left untreated or treated with just $10 \mathrm{ng} / \mathrm{mL}$ IFN- $\mathrm{\gamma}$ and

$910100 \mathrm{ng} / \mathrm{mL}$ AHT to induce expression of c-Myc. The cells were extracted, and metabolites

911 were analysed by LC-MS. Data are presented as mean \pm SD of triplicate wells. ${ }^{*}$ indicates a

$912 \mathrm{p}$ value $<0.01,{ }^{* * *}$ indicates a $\mathrm{p}$ value $<0.001$. The intracellular levels of tryptophan are

913 shown. e. WII-U2OS cells were either left uninfected or infected with Ctr at MOI 1 for $30 \mathrm{~h}$.

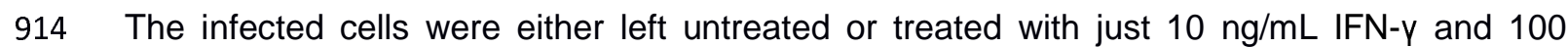

$915 \mathrm{ng} / \mathrm{mL}$ AHT to induce over expression of c-Myc. The media in which the cells were grown

916 was extracted and metabolites were analysed by LC-MS. Data are presented as mean \pm SD

917 of triplicate wells. ${ }^{* *}$ indicates a p value $<0.01,{ }^{* * *}$ indicates a $p$ value $<0.001$. The levels of

918 tryptophan present in medium is shown.

919 Figure 6: TCA intermediates and nucleosides can overcome IFN-y-induced 920 persistence.

921 a. A Heatmap with hierarchical clustering of all metabolites detected by LC-MS analysis of

922 (Fig. 5d). b/c. WII-U2OS cells were either left uninfected or infected with Ctr at MOI 1 for 30

$923 \mathrm{~h}$. The infected cells were either left untreated or treated with just $10 \mathrm{ng} / \mathrm{mL}$ IFN- $\gamma$ and 100

$924 \mathrm{ng} / \mathrm{mL}$ AHT to induce over expression of c-Myc. The cells were extracted, and metabolites

925 were analysed by LC-MS. Data are presented as mean \pm SD of triplicate wells. Intracellular

926 levels of metabolites like citrate, aconitate, $\alpha$-ketoglutarate, glutamate (b), nucleosides

927 (adenosine, guanosine, cytidine and uridine) (c) were determined and quantified. * indicates

928 a p value $<0.05,{ }^{* *}$ indicates a p value $<0.01,{ }^{* * *}$ indicates a $p$ value $<0.001,{ }^{* * * *}$ indicates a

$929 \mathrm{p}$ value $<0.0001$. d. Cells were left untreated or were pre-treated for $2 \mathrm{~h}$ with $10 \mathrm{ng} / \mathrm{mL}$ IFN-

$930 \mathrm{~V}, 4 \mathrm{mM}$ a-ketoglutarate (DMKG) or $100 \mu \mathrm{M}$ nucleosides, infected with Ctr at MOI 1 and lysed

931 at 30 hpi for Western blot analysis $(n=3)$. The a-ketoglutarate was supplied as cell-permeable

932 dimethyl ester. e. For the same culture conditions as in (d), an infectivity assay was 
933 performed. The cells were lysed at $48 \mathrm{hpi}$ and dilutions of the resulting Chlamydia containing

934 supernatant were added onto freshly plated WII-U2OS cells, which were then lysed at $30 \mathrm{hpi}$

935 to examine infectious progenies via Western blotting $(n=3)$. f. HeLa 229 cells were either

936 infected with Chlamydia or infected and treated with IFN-y and nucleosides (uridine/cytosine

937 or adenosine/guanosine) were added. The cells were lysed at $48 \mathrm{hpi}$ and dilutions of the

938 resulting Chlamydia containing supernatant were added onto freshly plated HeLa 229 cells,

939 which were then lysed at 30 hpi to examine infectious progenies via Western blot $(n=3)$.

Extended data Figure 1: IFN-y induces c-Myc depletion and impairs chlamydial growth.

a. An infectivity assay was performed in HeLa 229 cells to test for infectious progenies after addition of IFN-Y (10 ng/mL) or penicillin (1 unit). Lysate were subjected to Western blot analysis and bacterial load (cHSP60) was quantified by normalizing to B-Actin levels. b/c. HeLa 229 cells/ human Fimb cells were treated with different doses of IFN-y (10-50 ng/mL) and either left uninfected or infected with Ctr for 30/24 h. The cells were lysed and analysed via Western blotting $(n=3)$. d. HeLa 229 cells were treated with 10 or $50 \mathrm{ng} / \mathrm{mL}$ of IFN- $\mathrm{\gamma}$ and infected with Ctr D serovar. After $48 \mathrm{~h}$ the cells were lysed with glass beads and the supernatant was used to infect freshly plated HeLa 229 cells. After $30 \mathrm{~h}$ the cells were lysed and analysed via Western blotting. Bacterial load was determined by quantifying cHSP60 levels and B-Actin serves as loading control. e. Infectivity assay of the experiment shown in (Fig. 1g). The cells were lysed at $48 \mathrm{hpi}$ and dilutions of the resulting Chlamydia-containing supernatants were added onto fresh HeLa 229 cells, which were then lysed at $30 \mathrm{hpi}$ for Western blot analysis $(n=3)$. human organoids. 
959 were used to infect freshly plated HeLa 229 cells for an infectivity assay. Cells were fixed

960 with 4\% PFA after 30 hpi and immunostained for Ctr (cHSP60: green) and actin (Phalloidin:

961 red). The data from 5 different patients are presented here.

962 Extended data Figure 3: Expression of c-Myc rescues Chlamydia from persistence.

963 a. For the same culture conditions as in (Fig. 3a), an infectivity assay was performed. The 964 cells were lysed at $48 \mathrm{hpi}$ and dilutions of the resulting Chlamydia-containing supernatant 965 were added onto fresh HeLa 229 cells, which were then lysed at 30 hpi to examine infectious 966 progenies. The cHSP60 detect Chlamydia infection and the B-Actin serves as loading control $967(n=3)$. b. Human fallopian tube organoids were transduced with either lentivirus expressing cMyc or GFP as control (see Methods). The puromycin selected organoids where lysed and analysed via Western blotting. B-Actin serves as loading control. c/d. HeLa 229 cells were transduced with lentivirus expressing c-Myc (c) or GFP (d). The cells were analysed by Western blotting. B-Actin serves as the loading control. e. The virus preparations from (c) and (d) were used to infect human organoids derived from fallopian tube. The organoids were selected for puromycin. The images show organoids under selection 2, 4 and 6 days 974 respectively.

Extended data Figure 4: L-tryptophan activates the pGSK3ß-c-Myc axis and rescues chlamydial infection.

a. HeLa 229 cells were either left uninfected or infected with Chlamydia (Ctr) and treated with IFN-y with or without L-tryptophan (W). After $30 \mathrm{hpi}$, the cells were analysed via Western blotting ( $\mathrm{n}=3)$. b. HeLa 229 cells were infected with Ctr at MOI 1 for $24 \mathrm{~h}$ and or treated with IFN-y and L-tryptophan (W). The cells were lysed, and the supernatant was used to infect freshly plated HeLa 229 cells and bacterial load was determined by Western blotting for cHSP60. c. HeLa 229 cells were either left untreated or were treated with different amounts of L-tryptophan (W). After $24 \mathrm{~h}$ the cells were lysed and analysed by Western blotting. $\mathbf{d}$. HeLa 229 cells were infected with Ctr at MOI 1 for $30 \mathrm{~h}$ and treated with IFN-y or indole. The 
986 cells from (d) were lysed and the supernatant was used to infect freshly plated HeLa 229

987 cells to assess the infectivity of the progeny. f. HeLa 229 cells with AHT-inducible expression

988 of shc-Myc cells were treated with $1 \mathrm{ng} / \mathrm{mL}$ AHT for $2 \mathrm{~h}$. Cells were left untreated or were 989 pre-treated for $2 \mathrm{~h}$ with $100 \mathrm{ng} / \mathrm{mL}$ L-tryptophan (W), infected with Ctr at MOI 1 . The cells 990 were lysed, the supernatant was used to infect fresh cells, and lysed $30 \mathrm{hpi}$ to study 991 infectivity. g. WII-U2OS cells with inducible expression of c-Myc were left untreated or 992 treated with $100 \mathrm{ng} / \mathrm{mL}$ AHT and $10 \mathrm{ng} / \mathrm{mL}$ IFN-y for $2 \mathrm{~h}$. Cells were grown without L993 tryptophan, and infected with Ctr at MOI 1 and lysed at 30 hpi for Western blot analysis. $\mathbf{h}$. 994 Cells from the experiment shown in (g) were used for infectivity assay. i. Cells from human 995 fimbriae (human Fimb) were either left uninfected or infected with Ctr and treated with IFN-Y 996 with or without L-tryptophan (W). The cells were analysed by Western blotting 24 hpi. 997 cHSP60 shows the intensity of chlamydial infection and B-Actin serves as loading control $998(n=3)$.

\section{Extended data Figure 5: Pathway analysis}

1000

1001

1002

1003

1004

1005

1006

1007

1008

1009

1010

1011

1012

a.-d. WII-U2OS cells were either left uninfected or infected with Ctr at MOI 1 for $30 \mathrm{~h}$. The infected cells were either left untreated or treated with just $10 \mathrm{ng} / \mathrm{mL}$ IFN- $y$ and $100 \mathrm{ng} / \mathrm{mL}$ AHT to induce expression of c-Myc. The cells were extracted, and metabolites were analysed by LC-MS. Quality controls and data normalization were performed and a principal component analysis (PCA) (a), and pathway analysis (control vs infected (b), infected vs infected IFN- $\gamma$ treated $(\mathbf{c})$, infected IFN- $\gamma$ treated vs infected IFN- $\gamma$ treated and c-Myc expressed $(\mathbf{d}))$ are shown here. FDR $<0.05$, Impact $>0.4$.

\section{Extended data Figure 6: Urea cycle intermediates.}

a. Cartoon depicting TCA cycle and nucleotide biosynthesis in human cells. b. WII-U2OS cells were either left uninfected or infected with Ctr at MOI 1 for $30 \mathrm{~h}$. The infected cells were either left untreated or treated with just $10 \mathrm{ng} / \mathrm{mL}$ IFN-y and $100 \mathrm{ng} / \mathrm{mL}$ AHT to induce over expression of c-Myc. The cells were extracted, and metabolites were analysed by LC-MS. Data are presented as mean $\pm S D$ of triplicate wells. ${ }^{*}$ indicates a $p$ value $<0.05,{ }^{* *}$ indicates 
1013 a $p$ value $<0.01,{ }^{* * *}$ indicates a $p$ value $<0.001$ and ${ }^{* \star * *}$ indicates a $p$ value $<0.0001$. The 1014 levels of aspartate, serine and glycine are shown. c. Cartoon depicting the flux of aspartate 1015 from the TCA cycle. Oxaloacetate from TCA is converted into aspartate by mitochondrial 1016 malate dehydrogenase. The glutamate-aspartate antiporter transports aspartate into the 1017 cytosol where it is shuttled into the urea cycle. d-f. WII-U2OS cells were either left uninfected 1018 or infected with Ctr at MOI 1 for $30 \mathrm{~h}$. The infected cells were either left untreated or treated 1019 with just $10 \mathrm{ng} / \mathrm{mL}$ IFN-y and $100 \mathrm{ng} / \mathrm{mL}$ AHT to induce over expression of c-Myc. The cells 1020 were extracted, and metabolites were analysed by LC-MS. Data are presented as mean \pm 1021 SD of triplicate wells. * indicates a $p$ value $<0.05,{ }^{* *}$ indicates a p value $<0.01,{ }^{* * *}$ indicates 1022 a $p$ value $<0.001$ and ${ }^{* * * *}$ indicates a $p$ value $<0.0001$. The levels of arginine, ornithine, 1023 citrulline, nucleotide triphosphate (ATP, GTP, CTP and UTP) (e), and nucleotide 1024 monophosphate (AMP, GMP, CMP and UMP) (f) are shown. 
Fig. 1

a

b

1: Control

IFN-Y

doi: httpst//Gtiorg/10.1101/2022:03609p433696; this version postellellarch-9, 2021. The copyrighttholder for this preprint-

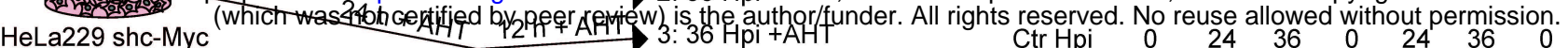

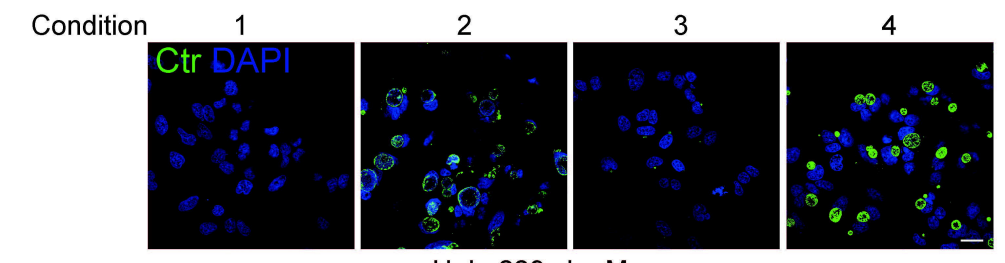

HeLa229 shc-Myc

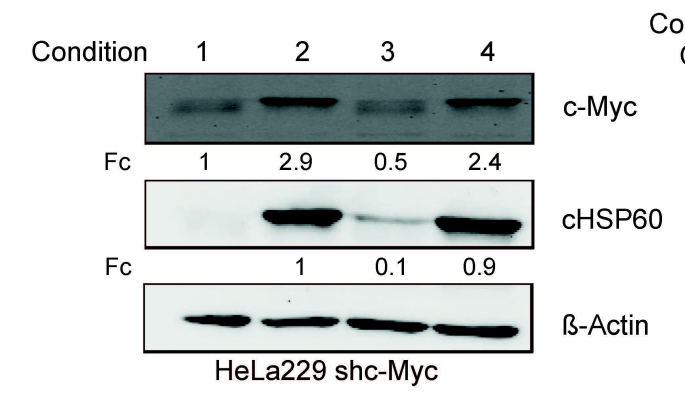

C

$\begin{array}{lllll}\text { IFN-y } & - & - & + & +\end{array}$

$\begin{array}{lllll}\text { Ctr Hpi } & 0 & 30 & 0 & 30\end{array}$
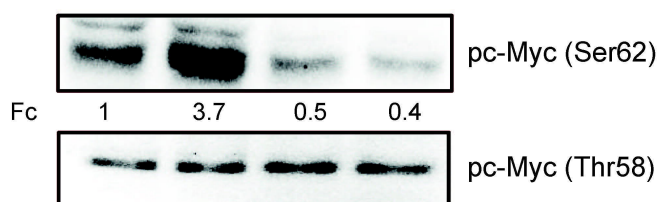

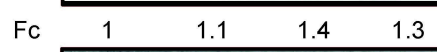

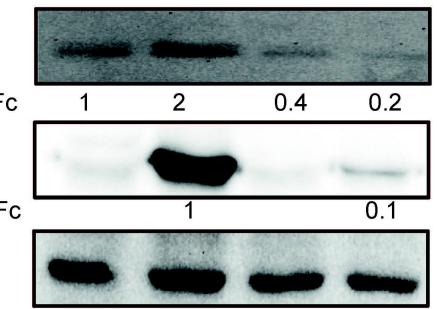

HeLa 229

f

IFN-y $\quad-\quad-\quad+\quad+$

$\begin{array}{lllll}\text { Ctr Hpi } & 0 & 30 & 0 & 30\end{array}$
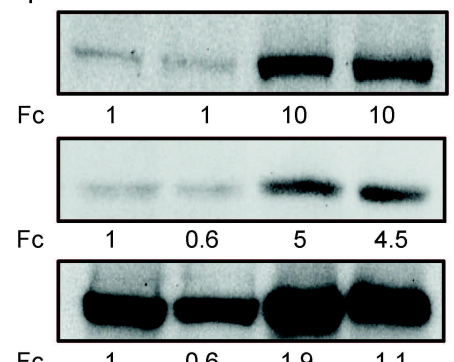

$\begin{array}{lllll}\text { Fc } & 1 & 0.6 & 1.9 & 1.1\end{array}$

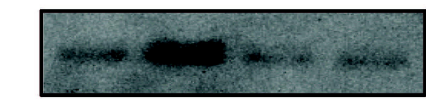

$\begin{array}{lllll}\text { FC } & 1 & 3.7 & 0.5 & 0.3\end{array}$

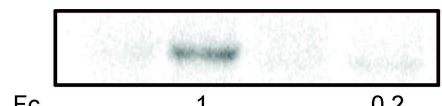

$\mathrm{Fc}$

0.2

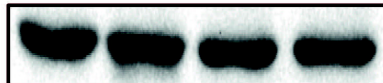

HeLa 229 $\underset{12 \mathrm{~h}-\mathrm{AHT}}{\longrightarrow} 4: 36 \mathrm{Hpi}-\mathrm{AHT}$

e

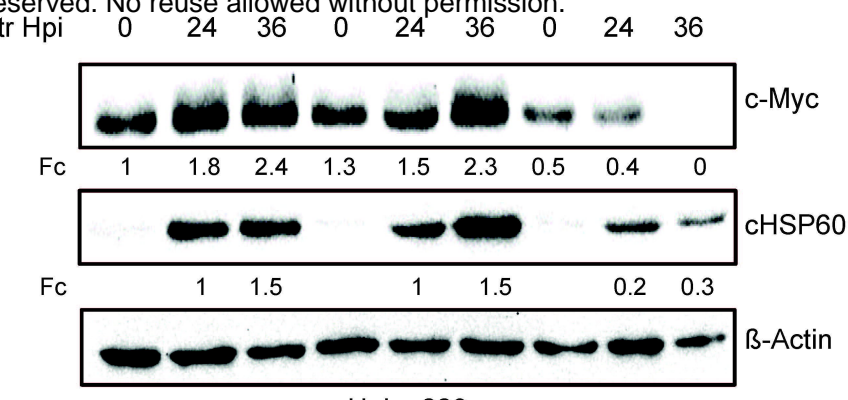

HeLa 229

d

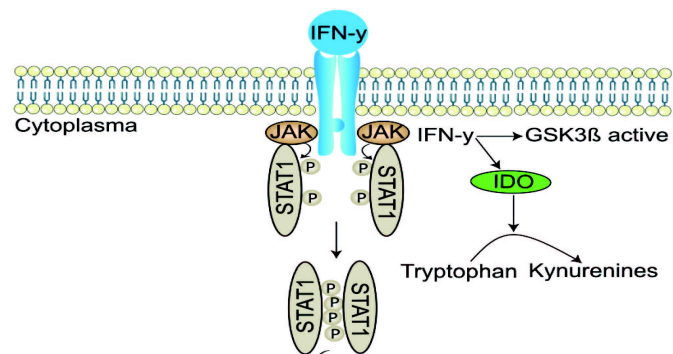

c-Myc

cHSP60

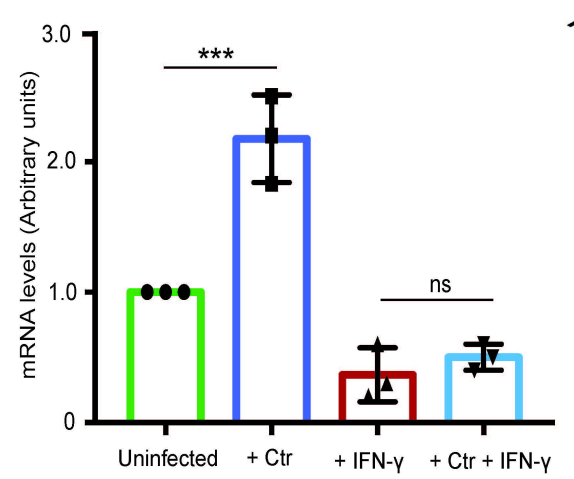

$\overbrace{\text { Nucleus }} \overbrace{\infty} \infty C$

这

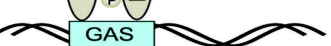

STAT1 inhibits c-myc
Activates IDO

\section{g}

$\begin{array}{rccccc}\text { STAT1 Si } & - & - & + & + & - \\ \text { IFN-Y } & - & - & - & + & + \\ \text { Ctr Hpi } & - & + & + & + & +\end{array}$

pStat1 Ser727

pStat1 Tyr701

Stat1
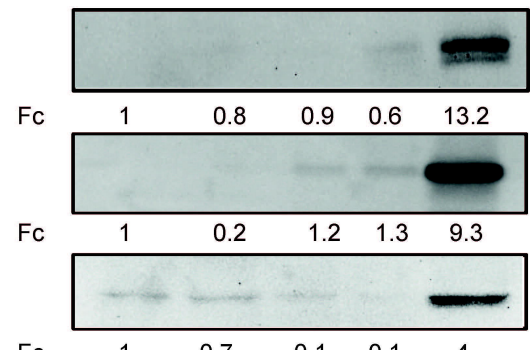

FC

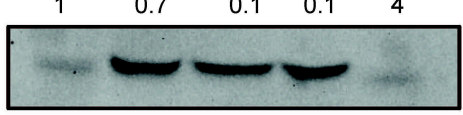

Fc

$\begin{array}{lll}6 & 5.2 & 0.3\end{array}$

cHSP60

ß Actin

HeLa 229 
Fig. 2

a

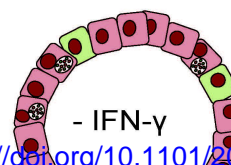

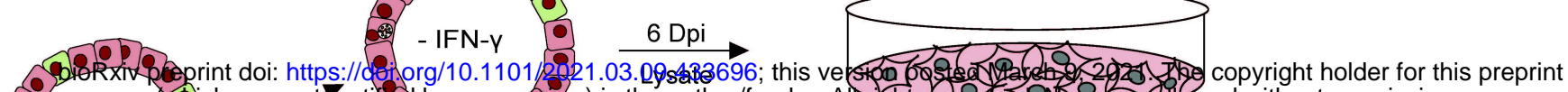

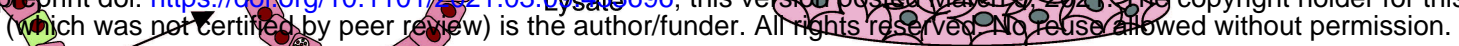

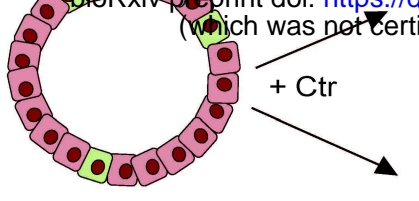

Human fallopian

tube organoids

800

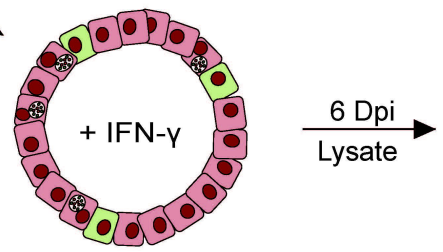

Primary infection

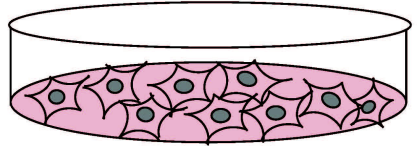

Secondary infection
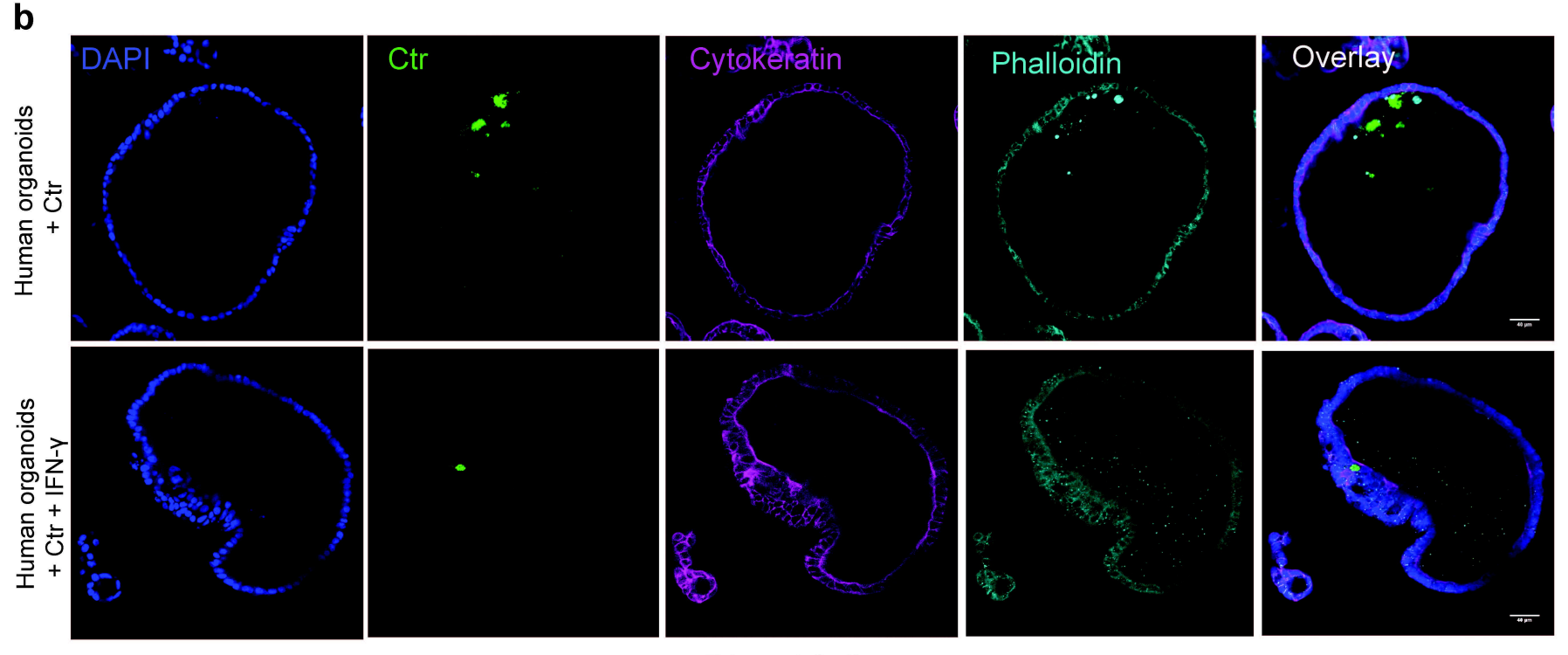

Primary infection

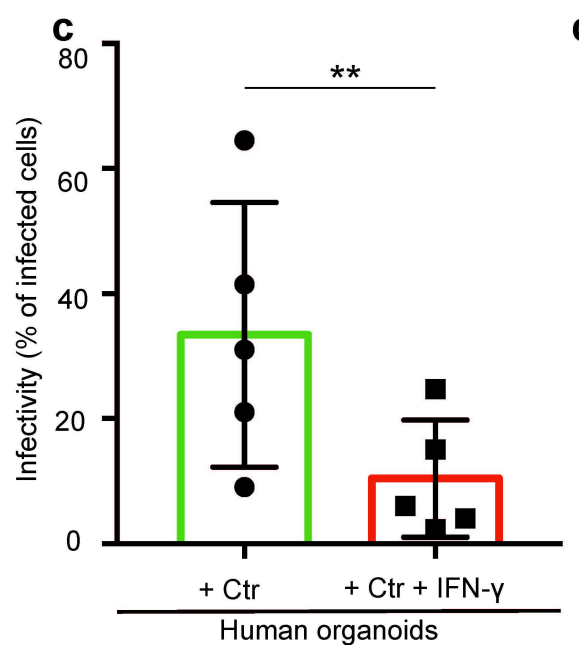

d

Human organoids

$+\mathrm{Ctr}$

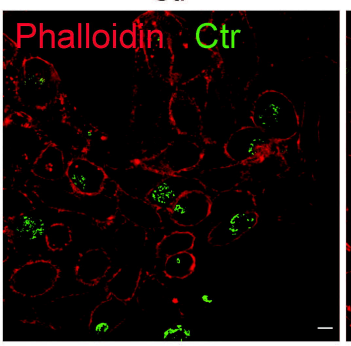

Secondary infection
Human organoids $+\mathrm{Ctr}+\mathrm{IFN}-\mathrm{Y}$

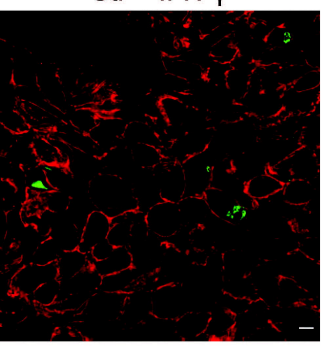

e
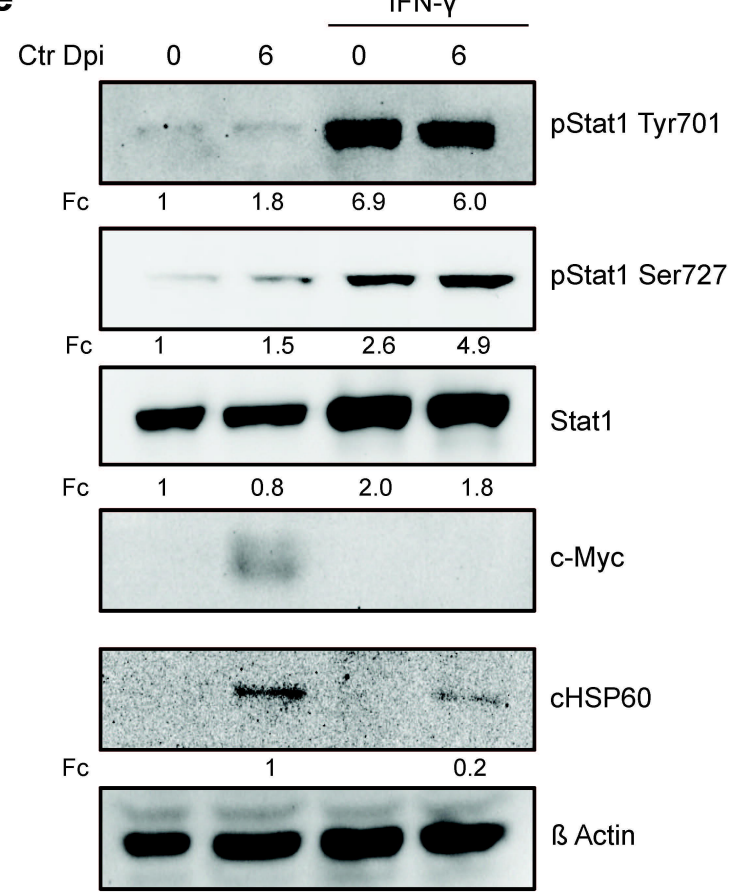

cHSP60

Human organoids 


\section{Fig. 3}

a

IFN-Y

AHT

Ctr Hpi

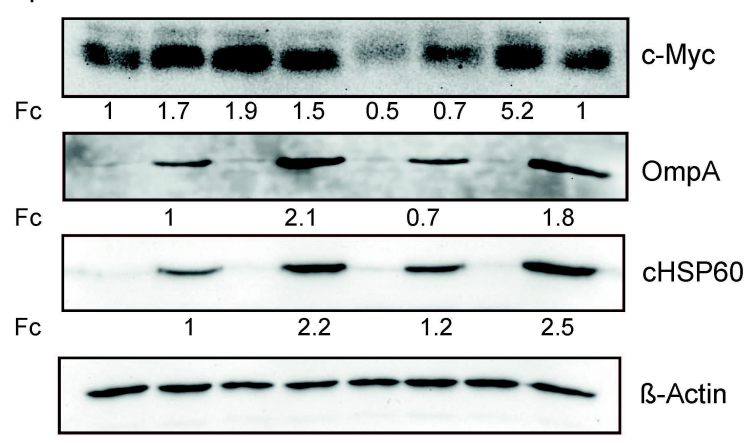

WII-U2OS

C
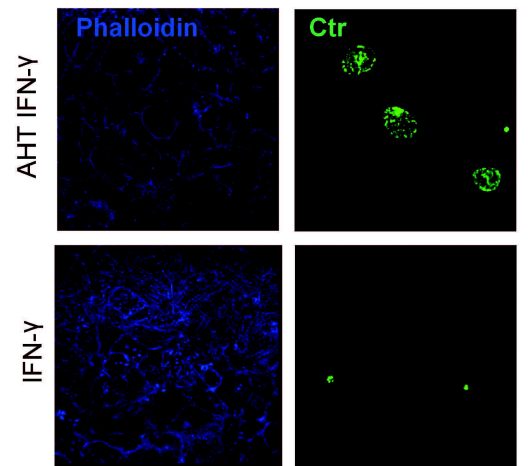

HeLa 229

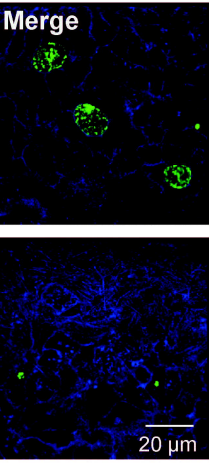

$\overline{20 \mu \mathrm{m}}$ b

1.0

2366; thi version posted March 9, 2021. The copyright holder for this preprint tBor/fund $\mathrm{r}$. All rights reserved. No reuse allowed without permission.

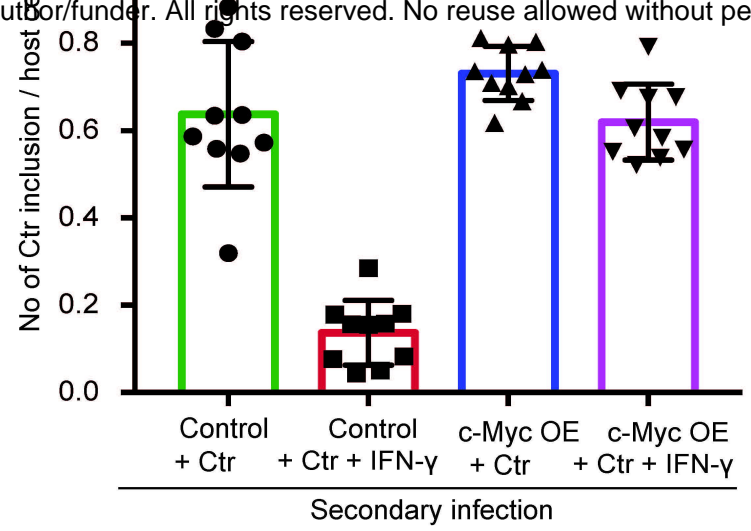

d

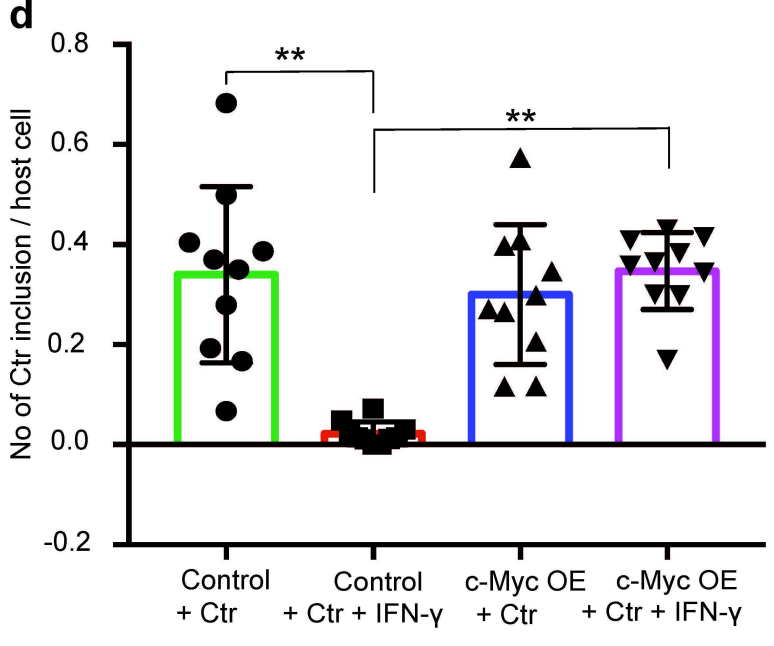

Human organoids

e

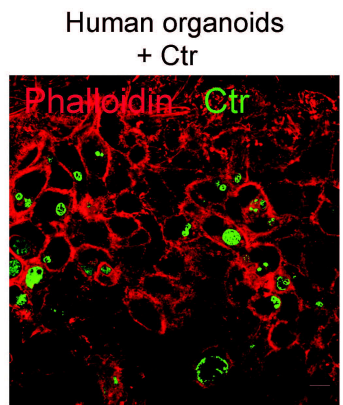

Human organoids $+\mathrm{Ctr}+\mathrm{IFN}-\mathrm{Y}$

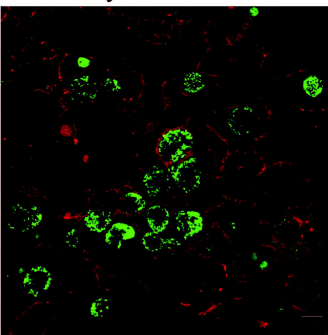

Human organoids c-Myc OE+ Ctr + IFN-y

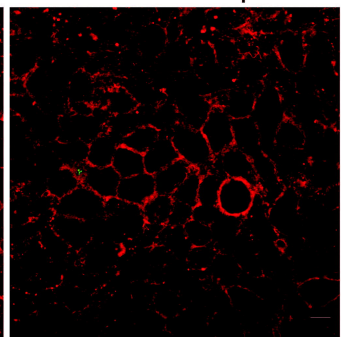

Secondary infection

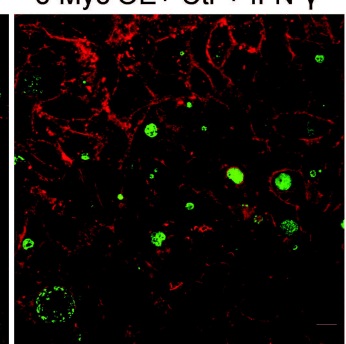


Fig. 4

$\mathbf{a}_{\mathrm{IFN}-\mathrm{Y}}-\quad+\quad+\quad+\quad+\quad+$

b

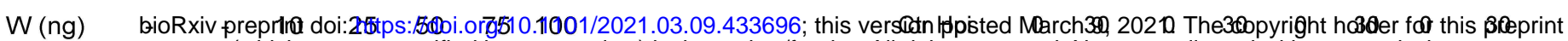

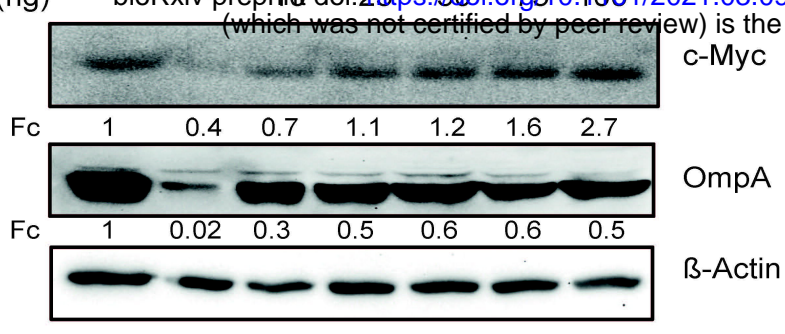

Secondary infection

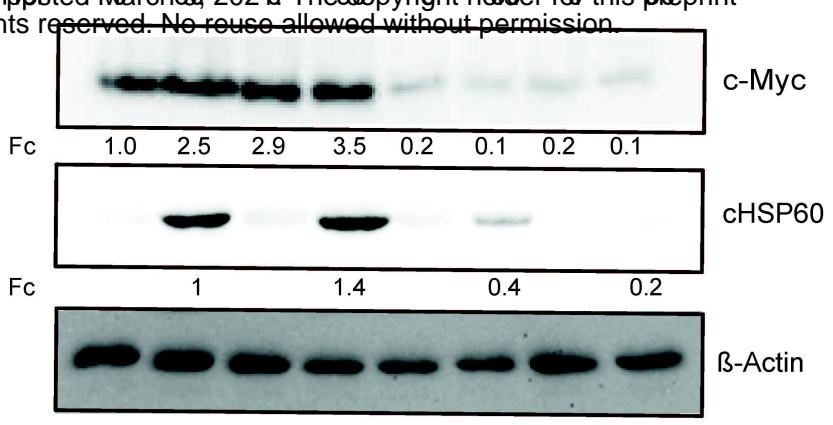

HeLa shc-Myc

\section{d}

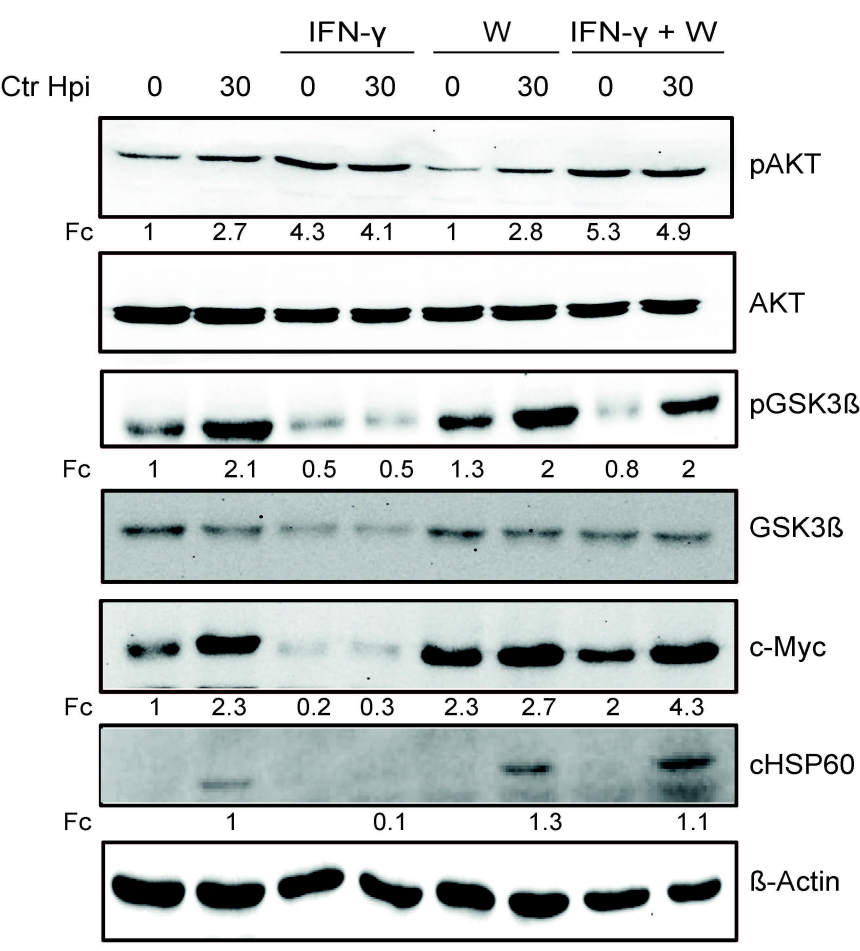

HeLa 229

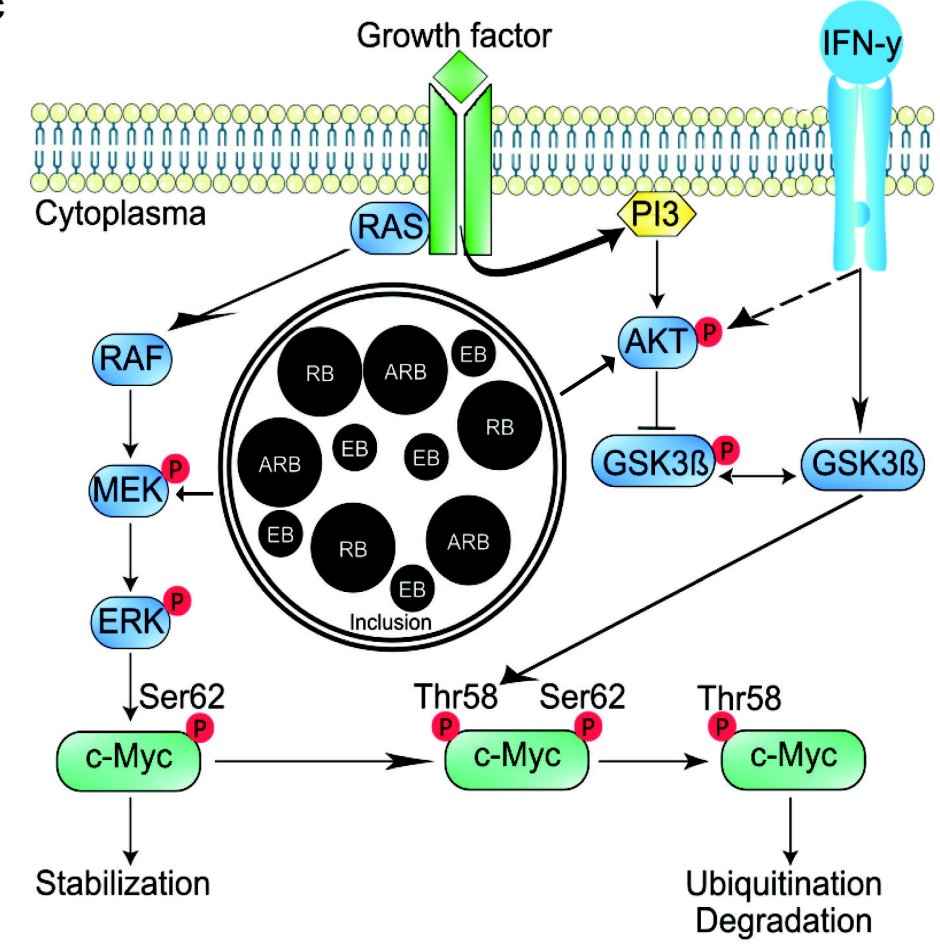


Fig. 5

a

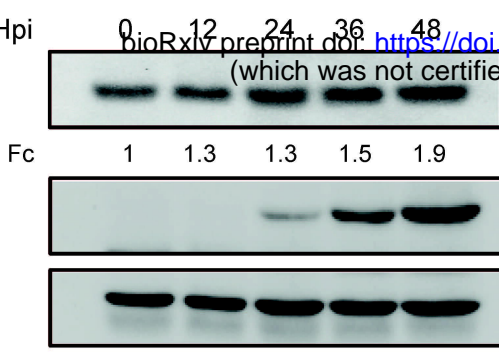

HeLa 229 b

IFN- $\mathrm{Y}$

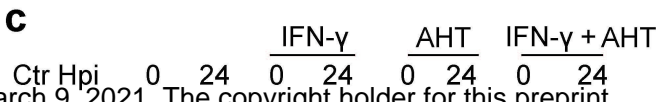

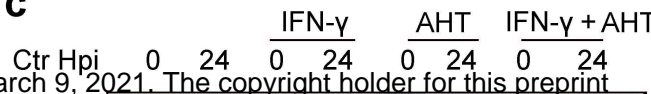

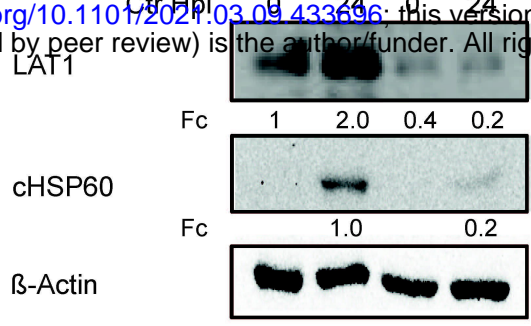

HeLa 229

\section{CHSP60}

ß-Actin

d

Tryptophan intracellular levles

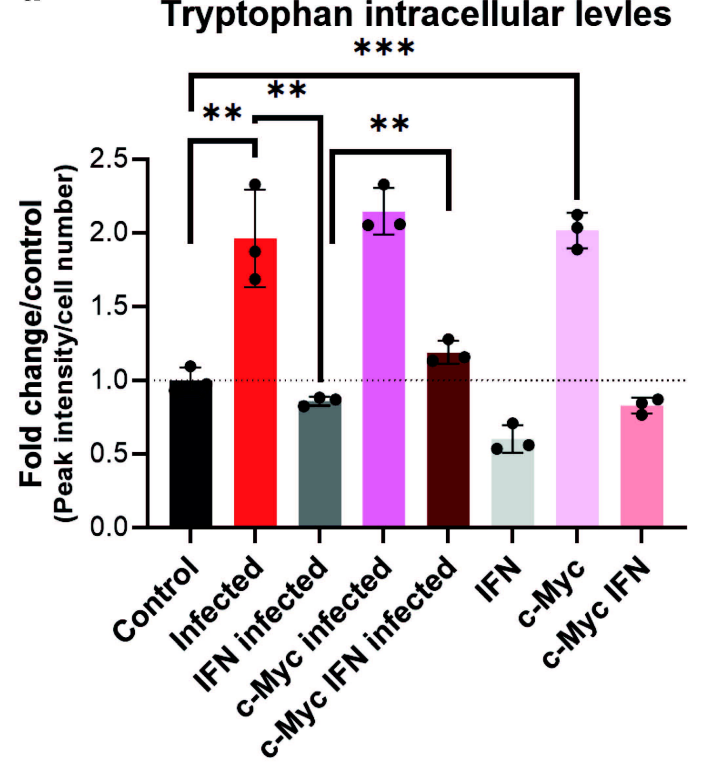

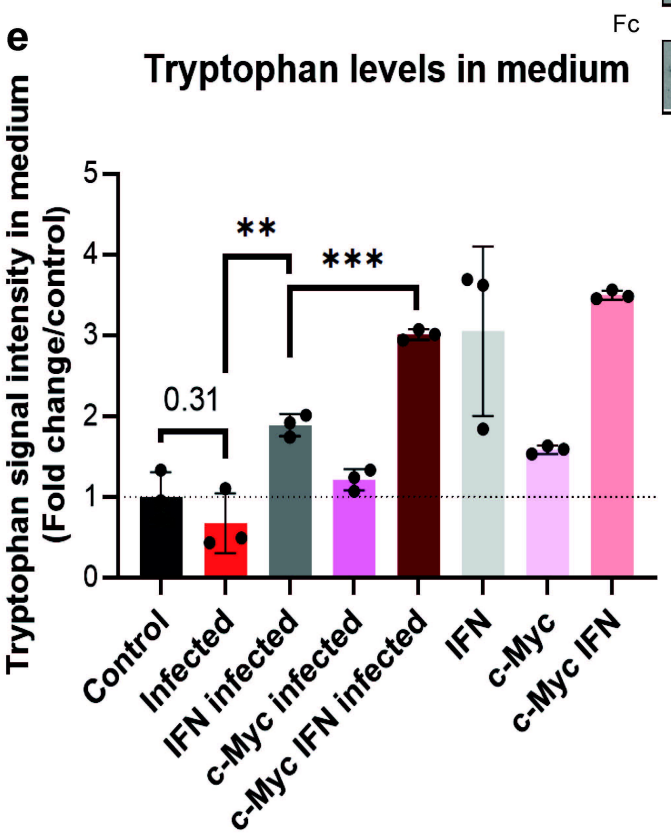

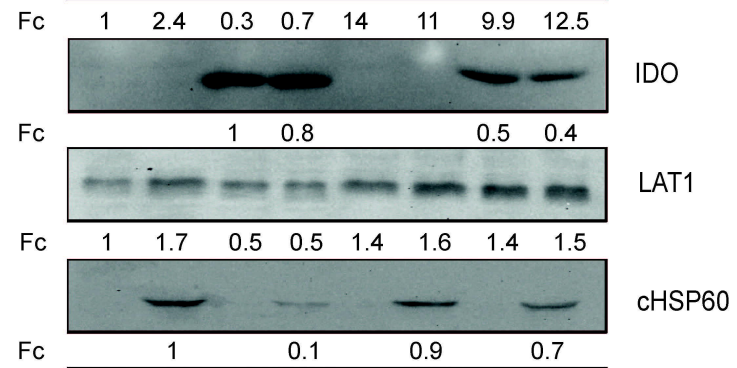

ß-Actin 
